\title{
Fermionic vacuum polarization in compactified cosmic string spacetime
}

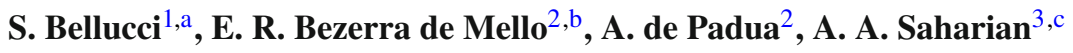 \\ ${ }^{1}$ INFN, Laboratori Nazionali di Frascati, Via Enrico Fermi 40, 00044 Frascati, Italy \\ ${ }^{2}$ Departamento de Física, Universidade Federal da Paraíba, Caixa Postal 5.008, 58.059-970 João Pessoa, PB, Brazil \\ ${ }^{3}$ Department of Physics, Yerevan State University, 1 Alex Manoogian Street, 0025 Yerevan, Armenia
}

Received: 25 November 2013 / Accepted: 2 December 2013 / Published online: 23 January 2014

(C) The Author(s) 2014. This article is published with open access at Springerlink.com

\begin{abstract}
We investigate the fermionic condensate and the vacuum expectation value (VEV) of the energy-momentum tensor for a charged massive fermionic field in the geometry of a cosmic string compactified along its axis. In addition, we assume the presence of two types of magnetic fluxes: a flux running along the cosmic string and another enclosed by the compact dimension. These fluxes give rise to AharanovBohm-like effects on the VEVs. The VEVs are decomposed into two parts corresponding to the geometry of a straight cosmic string without compactification plus a topological part induced by the compactification of the string axis. Both contributions are even periodic functions of the magnetic fluxes with period equal to the flux quantum. The vacuum energy density is equal to the radial stress for the parts corresponding to the straight cosmic string and the topological one. Moreover, the axial stress is equal to the energy density for the parts corresponding to the straight cosmic string; however, for massive fermionic fields this does not occur for the topological contributions. With respect to the dependence on the magnetic fluxes, both the fermionic condensate and the vacuum energy density, can be either positive or negative. Moreover, for points near the string, the main contribution to the VEVs comes from the straight cosmic string part, whereas at large distances the topological ones dominate. In addition to the local characteristics of the vacuum state, we also evaluate the part in the topological Casimir energy induced by the string.
\end{abstract}

\section{Introduction}

Topological and geometrical concepts are of great significance in recent developments of many areas of physics,

\footnotetext{
a e-mail: bellucci@lnf.infn.it

be-mail: emello@fisica.ufpb.br

ce-mail: saharian@ysu.am
}

including condensed matter physics, gauge field theories, and cosmology. In particular, the global properties of the spacetime manifold play an important role in quantum field theory. Several interesting quantum effects arise from the nontrivial topological structure of the background spacetime. A wellknown example of such quantum phenomena is the topological Casimir effect (for reviews see [1-5]). This effect is among the most striking macroscopic manifestations of the quantum properties of the vacuum state. The periodicity conditions imposed on a quantum field due to a nontrivial topology lead to a modification of the spectrum of zero-point fluctuations and result in shifting of the vacuum expectation values (VEVs) for physical quantities. In Kaluza-Klein type models, the dependence of the vacuum energy on the lengths of the extra dimensions can serve as a mechanism for the stabilization of the moduli fields. More recently, the topological Casimir effect has also been considered as a model for dark energy driving the accelerated expansion of the Universe [6-14]. The topological Casimir effect in cylindrical and toroidal carbon nanotubes is investigated in $[15,16]$ within the framework of a Dirac-like theory for the electronic states in graphene. In the present paper we investigate the interplay of quantum topological effects for a charged massive fermionic field coming from two different sources: from the conical geometry of a cosmic string spacetime and from the compactification of the string axis. Fermionic currents in this geometry, induced by magnetic fluxes, have been recently studied in [17]. The topological Casimir densities for a scalar field in compactified cosmic string spacetime are discussed in [18]. In this way, the present paper is a natural extension of the investigations started in these references.

Cosmic strings are linear topological defects which play an important role in cosmology. In the context of most unified particle physics models, these objects may have been created by phase transitions in the very early Universe [19,20]. Topological defects of similar structure arise in a number 
of condensed matter systems. Among the most important gravitational effects of cosmic strings is the generation of a scale-invariant spectrum of cosmological perturbations and, initially, cosmic strings have been considered as an alternative to inflation for generating primordial density perturbations from which galaxies grew. Though the observational evidence for acoustic oscillations in the angular power spectrum of cosmic microwave background has ruled out cosmic strings as the dominant source for primordial density perturbations, they are still candidates for the generation of a number of interesting physical effects such as gamma ray bursts [21], gravitational waves [22], and high-energy cosmic rays [23]. Moreover, fundamental string theory predicts the existence of macroscopic defects such as cosmic strings [24-27].

At large distances from the cosmic string core, the spacetime geometry for an infinite straight cosmic string has a conical topology with a planar angle deficit proportional to the linear mass density. The vacuum polarization effects in quantum field theory induced by this conical structure have been considered in a large number of papers. In the specific analysis for the VEV of the energy-momentum tensor, explicit calculations have been developed associated with scalar, fermionic, and electromagnetic fields [28-46]. The Casimir-Polder forces acting on a polarizable microparticle in the geometry of a cosmic string have been investigated in [47-49]. For charged fields, considering the presence of a magnetic flux running along the cosmic strings, there appear additional contributions to the corresponding vacuum polarization effects [32,50-55]. The magnetic flux along the cosmic string also induces vacuum current densities. This phenomenon has been investigated for scalar fields in [56,57]. The analysis of the induced fermionic currents in higherdimensional cosmic string spacetime in the presence of a magnetic flux has been developed in [58]. In this analysis the authors have shown that induced vacuum current densities along the azimuthal direction appear if the ratio of the magnetic flux by the quantum one has a nonzero fractional part. Moreover, the fermionic current induced by a magnetic flux in a $(2+1)$-dimensional conical spacetime and in the presence of a circular boundary has also been analyzed in [59] (for the combined effects of topology and boundaries on the quantum vacuum for scalar, electromagnetic, and fermionic fields in the geometry of a cosmic string see [60-71]).

The main objective of this paper is to investigate the combined effects of the planar angle deficit and of the compactification of the cosmic string axis on the fermionic condensate (FC) and on the VEV of the energy-momentum tensor. We assume the presence of a magnetic flux running along the string axis and the magnetic flux enclosed by the compact dimension. Although the corresponding operators are local, due to the global nature of the vacuum state, these VEVs carry important information as regards the global properties of the background spacetime. The FC plays an important role in models of dynamical breaking of the chiral symmetry. The VEV of the energy-momentum tensor acts as a source in the quasiclassical Einstein equations and is of key importance in modeling self-consistent dynamics involving fermionic fields.

The paper is organized as follows. In the next section we present the background geometry associated with the spacetime under consideration and provide the complete set of normalized positive- and negative-energy fermionic wave functions obeying quasiperiodic boundary condition along the string axis. By using the mode-summation procedure, we evaluate the FC. The condensate is decomposed into two terms: the first one corresponds to the geometry of a straight cosmic string with magnetic flux and the second term is induced by the compactification of the string axis. The latter is an even function of the magnetic fluxes, i.e., the flux along the string axis and the one enclosed by the string axis. We have provided closed expressions for both contributions. In Sect. 3, by using the mode-summation procedure, and we evaluate the VEVs for all components of the energymomentum tensor. Similar to the case of the FC, we provide a decomposition of these VEVs into the sum of a straight cosmic string and topological parts. Combined expressions for the components of the vacuum energy-momentum tensor and their asymptotics are presented in Sect. 4. In this section we also consider the part in the topological Casimir energy induced by the cosmic string and the magnetic flux. The most relevant conclusions of the paper are summarized in Sect. 5. Throughout the paper we use units with $G=\hbar=c=1$.

\section{Fermionic condensate}

\subsection{Mode functions}

In the presence of an external electromagnetic field with vector potential $A_{\mu}$, the quantum dynamics of a massive charged spinor field in curved spacetime is governed by the Dirac equation,

$i \gamma^{\mu} \mathcal{D}_{\mu} \psi-m \psi=0, \mathcal{D}_{\mu}=\partial_{\mu}+\Gamma_{\mu}+i e A_{\mu}$,

where $\gamma^{\mu}$ are the Dirac matrices in curved spacetime and $\Gamma_{\mu}$ is the spin connection. Both matrices are given in terms of the flat spacetime Dirac matrices, $\gamma^{(a)}$, by the relations

$\gamma^{\mu}=e_{(a)}^{\mu} \gamma^{(a)}, \Gamma_{\mu}=\frac{1}{4} \gamma^{(a)} \gamma^{(b)} e_{(a)}^{\nu} e_{(b) v ; \mu}$.

In (2.2), $e_{(a)}^{\mu}$ represents the tetrad basis satisfying the relation $e_{(a)}^{\mu} e_{(b)}^{v} \eta^{a b}=g^{\mu \nu}$, with $\eta^{a b}$ being the Minkowski spacetime metric tensor. 
The four-dimensional spacetime corresponding to an idealized cosmic string along the $z$-axis, can be written, by using cylindrical coordinates, by the line element:

$\mathrm{d} s^{2}=\mathrm{d} t^{2}-\mathrm{d} r^{2}-r^{2} \mathrm{~d} \phi^{2}-\mathrm{d} z^{2}$.

Here the coordinates take values in the ranges $r \geqslant 0,0 \leqslant$ $\phi \leqslant \phi_{0}=2 \pi / q,-\infty<t<+\infty$. The parameter $q$, greater than unity, is related to the linear mass density of the string, $\mu_{0}$, by $q^{-1}=1-4 \mu_{0}$. In the geometry described by (2.3) the gamma matrices can be taken in the form [17]

$\gamma^{0}=\gamma^{(0)}=\left(\begin{array}{ll}1 & 0 \\ 0 & -1\end{array}\right), \quad \gamma^{l}=\left(\begin{array}{ll}0 & \sigma^{l} \\ -\sigma^{l} & 0\end{array}\right)$,

where for the $2 \times 2$ matrices $\sigma^{l}, l=(r, \phi, z)$, one has

$$
\begin{aligned}
\sigma^{r} & =\left(\begin{array}{ll}
0 & e^{-i q \phi} \\
e^{i q \phi} & 0
\end{array}\right), \quad \sigma^{\phi}=-\frac{i}{r}\left(\begin{array}{ll}
0 & e^{-i q \phi} \\
-e^{i q \phi} & 0
\end{array}\right), \\
\sigma^{z} & =\left(\begin{array}{ll}
1 & 0 \\
0 & -1
\end{array}\right) .
\end{aligned}
$$

It is easy to check that with this choice the matrices (2.4) obey the Clifford algebra with the metric tensor from (2.3).

In the analysis that we want to develop, it will be assumed that the direction along the $z$-axis is compactified to a circle with length $L: 0 \leqslant z \leqslant L$. Along the compact dimension we impose the quasiperiodicity condition,

$\psi(t, r, \phi, z+L)=e^{2 \pi i \beta} \psi(t, r, \phi, z)$,

with a constant phase $\beta, 0 \leqslant \beta \leqslant 1$. In addition, we shall admit the existence of a gauge field with the constant vector potential

$A_{\mu}=\left(0,0, A_{\phi}, A_{z}\right)$.

The component $A_{\phi}$ is related to an infinitesimally thin magnetic flux, $\Phi_{\phi}$, running along the string by $A_{\phi}=$ $-q \Phi_{\phi} /(2 \pi)$ (note that $A_{\phi}$ and $A_{z}$ are the covariant components of the 4-vector $A_{\mu}=(0,-\mathbf{A})$ with $\mathbf{A}$ being the corresponding 3 -vector). Similarly, the axial component $A_{z}$ can be given in terms of the magnetic flux $\Phi_{z}$ enclosed by the $z$-axis as $A_{z}=-\Phi_{z} / L$. Though the magnetic field strength corresponding to (2.7) vanishes, the nontrivial topology of the background geometry leads to Aharonov-Bohm-like effects on the VEVs of physical observables.

In the present paper we are interested in the effects of the string compactification along its axis on the fermionic condensate (FC) and on the VEV of the energy-momentum tensor. For the evaluation of these VEVs, a complete set of fermionic mode functions is needed. In Ref. [17], we have shown that the positive- and negative-energy fermionic mode functions are uniquely specified by the set of quantum number $\sigma=(\lambda, k, j, s)$. These functions can be written in the form

$$
\begin{aligned}
\psi_{\sigma}^{( \pm)}(x)= & C_{\sigma}^{( \pm)} e^{\mp i E t+k z+i q(j-1 / 2) \phi} \\
& \times\left(\begin{array}{l}
J_{\beta_{j}}(\lambda r) \\
s J_{\beta_{j}+\epsilon_{j}}(\lambda r) e^{i q \phi} \\
\pm \frac{\tilde{k}_{l}-i s \epsilon_{j} \lambda}{E \pm m} J_{\beta_{j}}(\lambda r) \\
\mp s \frac{\tilde{k}_{l}-i s \lambda \epsilon_{j}}{E \pm m} J_{\beta_{j}+\epsilon_{j}}(\lambda r) e^{i q \phi}
\end{array}\right),
\end{aligned}
$$

where $J_{v}(x)$ is the Bessel function, $s= \pm 1, \lambda \geq 0$, and

$\beta_{j}=q|j+\alpha|-\epsilon_{j} / 2, \alpha=e A_{\phi} / q=-\Phi_{\phi} / \Phi_{0}$,

with $\epsilon_{j}=\operatorname{sgn}(j+\alpha)$ and with $\Phi_{0}=2 \pi / e$ being the flux quantum. The mode functions (2.8) are eigenfunctions for the projection of the total angular momentum operator along the cosmic string,

$\widehat{J}_{3} \psi_{\sigma}^{( \pm)}=\left(-i \partial_{\phi}+i \frac{q}{2} \gamma^{(1)} \gamma^{(2)}\right) \psi_{\sigma}^{( \pm)}=q j \psi_{\sigma}^{( \pm)}$,

with eigenvalues $j= \pm 1 / 2, \pm 3 / 2, \ldots$. The eigenvalues of the axial quantum number $k$ are determined by the periodicity condition (2.6),

$k=k_{l}=2 \pi(l+\beta) / L, \quad l=0, \pm 1, \pm 2, \ldots$.

The energy is expressed in terms of $\lambda$ and $l$ by the relation

$E=\sqrt{\lambda^{2}+\tilde{k}_{l}^{2}+m^{2}}, \tilde{k}_{l}=2 \pi(l+\tilde{\beta}) / L$,

where

$\tilde{\beta}=\beta+e A_{z} L /(2 \pi)=\beta-\Phi_{z} / \Phi_{0}$.

Note that, in order to simplify the representation of the mode functions, in the negative-energy modes we have changed the signs of the quantum numbers $(k, j, s)$, as compared with Ref. [17].

The constants $C_{\sigma}^{( \pm)}$in (2.8) are determined from the orthonormalization condition

$\int \mathrm{d}^{3} x \sqrt{\gamma}\left(\psi_{\sigma}^{(r)}\right)^{\dagger} \psi_{\sigma^{\prime}}^{\left(r^{\prime}\right)}=\delta_{\sigma \sigma^{\prime}} \delta_{r r^{\prime}}, r, r^{\prime}=+,-$,

where $\gamma$ is the determinant of the spatial metric tensor. The delta symbol on the right-hand side is understood as the Dirac delta function for continuous quantum numbers $(\lambda)$ and the Kronecker delta for discrete ones $(k, j, s, r)$. From (2.14) one finds

$\left|C_{\sigma}^{( \pm)}\right|^{2}=\frac{q \lambda(E \pm m)}{8 \pi L E}$. 
In deriving the mode functions (2.8) we have imposed the regularity condition on the string axis. A discussion of the contribution from irregular modes is given in [17].

The VEVs of physical observables will depend on $\beta$ and $\Phi_{z}$ in the combination given by (2.13). This result could be seen directly by making use of the gauge transformation $A_{\mu}=A_{\mu}^{\prime}+\partial_{\mu} \Lambda(x), \psi(x)=\psi^{\prime}(x) e^{-i e \Lambda(x)}$ with the function $\Lambda(x)=A_{z} z$. The new function $\psi^{\prime}(x)$ obeys the Dirac equation with $A_{z}^{\prime}=0$ and the periodicity condition $\psi^{\prime}(t, r, \phi, z+L)=e^{2 \pi i \tilde{\beta}} \psi^{\prime}(t, r, \phi, z)$. The VEVs are not changed under this gauge transformation and in the new gauge a single parameter $\tilde{\beta}$ appears instead of $\beta$ and $A_{z}$.

Having the complete set of wave functions we are in a position to evaluate the FC induced by the compactification of the string along its axis and also by the magnetic fluxes. The FC is defined as the VEV $\langle 0|\bar{\psi} \psi| 0\rangle \equiv\langle\bar{\psi} \psi\rangle$, where $|0\rangle$ corresponds to the vacuum state, and $\bar{\psi}=\psi^{\dagger} \gamma^{(0)}$ is the Dirac adjoint. Expanding the field operator in terms of the complete set $\left\{\psi_{\sigma}^{(+)}, \psi_{\sigma}^{(-)}\right\}$and by using the standard anticommutation relations for the annihilation and creation operators, the following formula for the FC is obtained:

$\langle\bar{\psi} \psi\rangle=\sum_{\sigma} \bar{\psi}_{\sigma}^{(-)} \psi_{\sigma}^{(-)}$,

where we use the compact notation defined by

$$
\sum_{\sigma}=\int_{0}^{\infty} \mathrm{d} \lambda \sum_{l=-\infty}^{+\infty} \sum_{s= \pm 1} \sum_{j= \pm 1 / 2, \ldots}
$$

This VEV is a periodic function of the fluxes $\Phi_{\phi}$ and $\Phi_{z}$ with period equal to the flux quantum. In particular, if we write the parameter $\alpha$ in (2.9) in the form

$\alpha=n_{0}+\alpha_{0},\left|\alpha_{0}\right|<1 / 2$,

where $n_{0}$ is an integer number, the FC will depend on $\alpha_{0}$ only. Note that, for the boundary condition at the cone apex used in [59], there are no square integrable irregular modes for $\left|\alpha_{0}\right| \leqslant(1-1 / q) / 2$.

Substituting the wave function (2.8) into (2.16), we can see that the terms with $s=1$ and $s=-1$ give the same contribution and one gets

$$
\langle\bar{\psi} \psi\rangle=-\frac{q m}{2 \pi L} \sum_{l=-\infty}^{+\infty} \sum_{j} \int_{0}^{\infty} \mathrm{d} \lambda \frac{\lambda}{E}\left[J_{\beta_{j}}^{2}(\lambda r)+J_{\beta_{j}+\epsilon_{j}}^{2}(\lambda r)\right] .
$$

In what follows we shall use the notation

$$
\sum_{j}=\sum_{j= \pm 1 / 2, \ldots}
$$

Of course, the expression in the right-hand side of (2.19) is divergent and a regularization is necessary. Here we assume the presence of a cutoff function without writing it explicitly. As we shall see, the specific form of this function is not relevant in the discussion below. By taking into account the expression (2.12) for the energy, the summation over the quantum number $l$ can be developed by using the Abel-Plana summation formula in the form [72] ${ }^{1}$

$$
\begin{aligned}
& \frac{2 \pi}{L} \sum_{l=-\infty}^{\infty} f\left(\left|\tilde{k}_{l}\right|\right) \\
& \quad=2 \int_{0}^{\infty} \mathrm{d} k f(k)+i \int_{0}^{\infty} \mathrm{d} k \sum_{\delta= \pm 1} \frac{f(i k)-f(-i k)}{e^{L k+2 \pi i \delta \tilde{\beta}}-1},
\end{aligned}
$$

taking $f(k)=\left(k^{2}+\lambda^{2}+m^{2}\right)^{-1 / 2}$. Consequently the FC can be written in the decomposed form

$\langle\bar{\psi} \psi\rangle=\langle\bar{\psi} \psi\rangle_{s}+\langle\bar{\psi} \psi\rangle_{c}$,

where $\langle\bar{\psi} \psi\rangle_{s}$ is the contribution due to the first integral in the right-hand side of (2.21) and corresponds to the FC in the geometry of the cosmic string spacetime in the absence of compactification. As to the term $\langle\bar{\psi} \psi\rangle_{c}$, it vanishes in the limit $L \rightarrow \infty$ and this contribution is induced by the compactification of the string along its axis.

In the absence of magnetic flux, corresponding to $\alpha_{0}=0$, a closed analytic expression for $\langle\bar{\psi} \psi\rangle_{s}$ is provided in [71]. In this special case an alternative integral representation is derived in [63]. The FC in a $(2+1)$-dimensional conical spacetime in the presence of a magnetic flux has been evaluated in [67] for a massive field obeying the MIT bag boundary condition on a circular boundary. To our knowledge, a closed expression for the FC in a four-dimensional cosmic string spacetime in the presence of magnetic flux running along the string has not been obtained yet. So in order to fulfill this blank, we shall include this calculation in this paper.

\subsection{FC in the geometry of straight cosmic string}

Combining (2.19) and (2.21), for the FC in the geometry of a straight cosmic string we get the integral representation

$$
\begin{aligned}
\langle\bar{\psi} \psi\rangle_{s}= & -\frac{q m}{2 \pi^{2}} \int_{0}^{\infty} \mathrm{d} \lambda \lambda \int_{0}^{\infty} \mathrm{d} k \frac{1}{\sqrt{k^{2}+\lambda^{2}+m^{2}}} \\
& \times \sum_{j}\left[J_{\beta_{j}}^{2}(\lambda r)+J_{\beta_{j}+\epsilon_{j}}^{2}(\lambda r)\right] .
\end{aligned}
$$

\footnotetext{
1 For generalizations of the Abel-Plana formula see [73].
} 
For the further transformation, we use the relation

$$
\frac{1}{\sqrt{k^{2}+\lambda^{2}+m^{2}}}=\frac{2}{\sqrt{\pi}} \int_{0}^{\infty} \mathrm{d} s e^{-\left(k^{2}+\lambda^{2}+m^{2}\right) s^{2}} .
$$

Substituting this into (2.23), we can easily integrate over the variable $k$. For the integral over $\lambda$, we use the integral involving the square of the Bessel function from [74] with the result

$$
\begin{aligned}
& \int_{0}^{\infty} \mathrm{d} \lambda \lambda e^{-s^{2} \lambda^{2}}\left[J_{\beta_{j}}^{2}(\lambda r)+J_{\beta_{j}+\epsilon_{j}}^{2}(\lambda r)\right] \\
& \quad=\frac{e^{-y}}{2 s^{2}}\left[I_{\beta_{j}}(y)+I_{\beta_{j}+\epsilon_{j}}(y)\right],
\end{aligned}
$$

with $y=r^{2} /\left(2 s^{2}\right)$ and with $I_{\nu}(z)$ being the modified Bessel function. As a result, the FC is represented in the form

$$
\langle\bar{\psi} \psi\rangle_{s}=-\frac{q m}{(2 \pi r)^{2}} \int_{0}^{\infty} \mathrm{d} y e^{-y-m^{2} r^{2} /(2 y)} \mathcal{J}\left(q, \alpha_{0}, y\right),
$$

where we have defined the function

$\mathcal{J}\left(q, \alpha_{0}, y\right)=\mathcal{I}\left(q, \alpha_{0}, y\right)+\mathcal{I}\left(q,-\alpha_{0}, y\right)$,

with $\mathcal{I}\left(q, \alpha_{0}, y\right)=\sum_{j} I_{\beta_{j}}(y)$ and $\mathcal{I}\left(q,-\alpha_{0}, y\right)=\sum_{j}$ $I_{\beta_{j}+\epsilon_{j}}(y)$.

An integral representation for the function $\mathcal{I}\left(q, \alpha_{0}, y\right)$, suitable for the extraction of the divergent part in the FC, is derived in [59]. By using that representation, for the function (2.27) one finds the following formula:

$$
\begin{aligned}
& \mathcal{J}\left(q, \alpha_{0}, y\right) \\
& =\frac{2}{q} e^{y}+\frac{4}{\pi} \int_{0}^{\infty} \mathrm{d} x \frac{h\left(q, \alpha_{0}, x\right) \sinh x}{\cosh (2 q x)-\cos (q \pi)} e^{-y \cosh (2 x)} \\
& \quad+\frac{4}{q} \sum_{k=1}^{p}(-1)^{k} \cos (\pi k / q) \cos \left(2 \pi k \alpha_{0}\right) e^{y \cos (2 \pi k / q)},
\end{aligned}
$$

where $p$ is an integer defined by $2 p \leqslant q<2 p+2$ and for $1 \leqslant q<2$ the last term on the right-hand side is absent. The function in the integrand of (2.28) is given by the expression

$$
\begin{aligned}
h\left(q, \alpha_{0}, x\right)= & \cos \left[q \pi\left(1 / 2+\alpha_{0}\right)\right] \sinh \left[\left(1-2 \alpha_{0}\right) q x\right] \\
& +\cos \left[q \pi\left(1 / 2-\alpha_{0}\right)\right] \sinh \left[\left(1+2 \alpha_{0}\right) q x\right] .
\end{aligned}
$$

Note that $\mathcal{J}\left(q, \alpha_{0}, y\right)$ is an even function of $\alpha_{0}$.
In the case of integer values of $q$ and for

$\alpha_{0}=\frac{1}{2}-\frac{n+1 / 2}{q}$,

with an integer $n$, one has $h\left(q, \alpha_{0}, x\right)=0$. From the condition $\left|\alpha_{0}\right|<1 / 2$ we find $0 \leqslant n<q-1 / 2$. In this case a simpler expression for the function $\mathcal{J}\left(q, \alpha_{0}, y\right)$ is obtained:

$$
\begin{aligned}
& \mathcal{J}\left(q, \alpha_{0}, y\right) \\
& =\frac{2}{q} \sum_{k=0}^{q-1} \cos (\pi k / q) \cos ((2 n+1) \pi k / q) e^{y \cos (2 \pi k / q)} .
\end{aligned}
$$

We see that the first term on the right-hand side of (2.28) provides a contribution to FC independent of $\alpha_{0}$ and $q$. It corresponds to the FC in Minkowski spacetime in the absence of magnetic flux. This term provides FC with a divergent result. Because the geometry of the cosmic string is flat outside the string core, the renormalization for $\langle\bar{\psi} \psi\rangle_{s}$ reduces; from this expression the Minkowski spacetime part is subtracted. So we discard the exponential term in (2.28). The other terms provide contributions to the FC due to the magnetic flux and nontrivial topology of the straight cosmic string. These terms are finite and do not require any renormalization procedure. So the cutoff function, assumed implicitly before, can be safely removed. Substituting (2.28) into (2.26), the integrals over the variable $y$ are evaluated with the help of the formula from [74], and the final result for the renormalized FC is written as

$$
\begin{aligned}
\langle\bar{\psi} \psi\rangle_{s}^{\text {ren }}= & -\frac{2 m^{3}}{\pi^{2}}\left[\sum_{k=1}^{p}(-1)^{k} \cos (\pi k / q) \cos \left(2 \pi k \alpha_{0}\right) f_{1}\left(2 m r s_{k}\right)\right. \\
& \left.+\frac{q}{\pi} \int_{0}^{\infty} \mathrm{d} x \frac{h\left(q, \alpha_{0}, x\right) \sinh x}{\cosh (2 q x)-\cos (q \pi)} f_{1}(2 m r \cosh x)\right] .
\end{aligned}
$$

Here we have introduced the notation

$$
f_{v}(x)=K_{v}(x) / x^{\nu}, s_{k}=\sin (\pi k / q),
$$

with $K_{v}(z)$ being the Macdonald function. In the absence of the magnetic flux one has $\alpha_{0}=0$ and, hence, $h(q, 0, x)=$ $2 \cos (q \pi / 2) \sinh (q x)$. In this case the formula (2.32) is reduced to the one derived in [71]. For $q=1$, Eq. (2.32) gives the FC induced by the magnetic flux in Minkowski spacetime. For the special case mentioned in (2.30), the integral term vanishes and the renormalized value for the $\mathrm{FC}$ is expressed by

$$
\langle\bar{\psi} \psi\rangle_{s}^{\mathrm{ren}}=-\frac{m^{3}}{\pi^{2}} \sum_{k=1}^{q-1} \cos (\pi k / q) \cos ((2 n+1) \pi k / q) f_{1}\left(2 m r s_{k}\right) .
$$




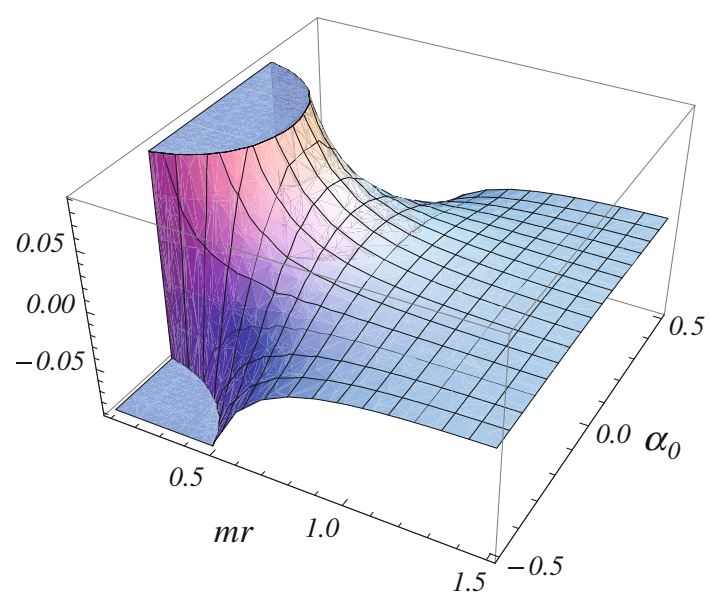

Fig. 1 FC in the geometry of a straight cosmic string, $\langle\bar{\psi} \psi\rangle_{s}^{\text {ren }} / \mathrm{m}^{3}$, versus the distance from the string and the parameter $\alpha_{0}$. For the parameter describing the planar angle deficit we have taken $q=2.5$

Let us consider some limiting cases. First of all, by using the asymptotic

$f_{v}(x) \sim 2^{v-1} \Gamma(v) x^{-2 v}, x \rightarrow 0$,

we see that the FC vanishes for a massless field. At large distances from the string, $m r \gg 1$, the FC is suppressed by the factor $e^{-2 m r}$ for $1 \leqslant q \leqslant 2$ and by the factor $e^{-2 m r \sin (\pi / q)}$ for $q>2$. Finally, on the string, $r \rightarrow 0$, the FC diverges as $1 / r^{2}$.

In Fig. 1 we have plotted the FC in the geometry of a straight cosmic string, $\langle\bar{\psi} \psi\rangle_{s}^{\text {ren }} / \mathrm{m}^{3}$, as a function of the distance from the string and of the parameter $\alpha_{0}$ characterizing the magnetic flux along the string. For the parameter describing the planar angle deficit we have taken the value $q=2.5$. As we see, in dependence of the flux, the FC can be either positive or negative. In the absence of magnetic flux the FC is positive.

\subsection{Topological part}

Let us now develop the calculation for the contribution to the FC induced by the compactification. This part comes from the second integral in (2.21) and is presented in the form

$$
\begin{aligned}
\langle\bar{\psi} \psi\rangle_{c}= & -\frac{q m}{2 \pi^{2}} \sum_{j} \int_{0}^{\infty} \mathrm{d} \lambda \lambda \int_{\sqrt{\lambda^{2}+m^{2}}}^{\infty} \mathrm{d} k \\
& \times \frac{J_{\beta_{j}}^{2}(\lambda r)+J_{\beta_{j}+\epsilon_{j}}^{2}(\lambda r)}{\sqrt{k^{2}-\lambda^{2}-m^{2}}} \sum_{\delta= \pm 1} \frac{1}{e^{L k+2 \delta \pi i \tilde{\beta}}-1} .
\end{aligned}
$$

To continue the calculation, in the integrand of (2.36) we use the series expansion

$$
\left(e^{u}-1\right)^{-1}=\sum_{l=1}^{\infty} e^{-l u}
$$

The integral over $k$ is expressed in terms of the function $K_{0}(x)$ and the above expression becomes

$$
\begin{aligned}
\langle\bar{\psi} \psi\rangle_{c}= & -\frac{q m}{\pi^{2}} \sum_{l=1}^{\infty} \cos (2 \pi l \tilde{\beta}) \int_{0}^{\infty} \mathrm{d} \lambda \lambda K_{0}\left(l L \sqrt{\lambda^{2}+m^{2}}\right) \\
& \times \sum_{j}\left[J_{\beta_{j}}^{2}(\lambda r)+J_{\beta_{j}+\epsilon_{j}}^{2}(\lambda r)\right] .
\end{aligned}
$$

By making use of the integral representation for the Macdonald function,

$K_{v}(x)=\frac{1}{2}\left(\frac{x}{2}\right)^{v} \int_{0}^{\infty} \mathrm{d} t \frac{e^{-t-x^{2} /(4 t)}}{t^{\nu+1}}$,

we see that the integral over $\lambda$ becomes of the form (2.25). Defining the new variable $y=2 t r^{2} /\left(l^{2} L^{2}\right)$, the above expression is written as

$$
\begin{aligned}
\langle\bar{\psi} \psi\rangle_{c}= & -\frac{q m}{2 \pi^{2} r^{2}} \sum_{l=1}^{\infty} \cos (2 \pi l \tilde{\beta}) \int_{0}^{\infty} \mathrm{d} y \\
& \times e^{-y\left[1+l^{2} L^{2} /\left(2 r^{2}\right)\right]-m^{2} r^{2} /(2 y)} \mathcal{J}\left(q, \alpha_{0}, y\right) .
\end{aligned}
$$

The integrand in this expression is nonnegative and, hence, the topological part in the $\mathrm{FC}$ is always negative for $\tilde{\beta}=0$. In this case $\left|\langle\bar{\psi} \psi\rangle_{c}\right|$ is a monotonically decreasing function of $L$. Substituting the expression (2.28) into (2.40) and integrating over $y$ we get the expression

$$
\begin{aligned}
\langle\bar{\psi} \psi\rangle_{c}= & -\frac{4 m^{3}}{\pi^{2}} \sum_{l=1}^{\infty} \cos (2 \pi l \tilde{\beta}) \\
& \times\left[\sum_{k=0}^{p}(-1)^{k} \cos (\pi k / q) \cos \left(2 \pi k \alpha_{0}\right) f_{1}\left(m L \sqrt{l^{2}+\rho_{k}^{2}}\right)\right. \\
& +\frac{q}{\pi} \int_{0}^{\infty} \mathrm{d} x \frac{h\left(q, \alpha_{0}, x\right) \sinh x}{\cosh (2 q x)-\cos (q \pi)} f_{1}\left(m L \sqrt{\left.l^{2}+\eta^{2}(x)\right)}\right],
\end{aligned}
$$

where we have introduced the notation

$\rho_{k}=\frac{2 r}{L} \sin (\pi k / q), \eta(x)=\frac{2 r}{L} \cosh x$.

In (2.41), the prime on the summation over $k$ means that the term with $k=0$ should be taken with the weight $1 / 2$. For a massless field the topological part vanishes. In the special case defined by (2.30), the topological part in the FC reads

$$
\begin{aligned}
\langle\bar{\psi} \psi\rangle_{c}= & -\frac{2 m^{3}}{\pi^{2}} \sum_{l=1}^{\infty} \cos (2 \pi l \tilde{\beta}) \sum_{k=0}^{q-1} \cos (\pi k / q) \\
& \times \cos ((2 n+1) \pi k / q) f_{1}\left(m L \sqrt{l^{2}+\rho_{k}^{2}}\right) .
\end{aligned}
$$


In the absence of a cosmic string and of flux along the $z$-axis one has $q=1, \alpha_{0}=0$, and in (2.41) the term $k=0$ survives only, with the result

$$
\langle\bar{\psi} \psi\rangle_{c}^{(0)}=-\frac{2 m^{3}}{\pi^{2}} \sum_{l=1}^{\infty} \cos (2 \pi l \tilde{\beta}) f_{1}(m L l) .
$$

This quantity corresponds to the FC in Minkowski spacetime with spatial topology $R^{2} \times S^{1}$ and with the length of the compact dimension $L$. The FC for a more general case of spatial topology $R^{p} \times\left(S^{1}\right)^{D-p}$ has been investigated in [15]. The topological part ( 2.41) is finite on the string:

$$
\langle\bar{\psi} \psi\rangle_{c}=\left[1+2 s_{0}\left(q, \alpha_{0}\right)\right]\langle\bar{\psi} \psi\rangle_{c}^{(0)}, \quad r=0,
$$

with $\langle\bar{\psi} \psi\rangle_{c}^{(0)}$ given by (2.44) and with the notation

$$
\begin{aligned}
s_{n}\left(q, \alpha_{0}\right)= & \sum_{k=1}^{p} \frac{(-1)^{k}}{s_{k}^{n}} \cos (\pi k / q) \cos \left(2 \pi k \alpha_{0}\right) \\
& +\frac{q}{\pi} \int_{0}^{\infty} \mathrm{d} x \frac{h\left(q, \alpha_{0}, x\right) \sinh x \cosh ^{-n} x}{\cosh (2 q x)-\cos (q \pi)} .
\end{aligned}
$$

Hence, near the string the FC is dominated by the part $\langle\bar{\psi} \psi\rangle_{s}$. In the absence of magnetic flux, for the function (2.46) we have

$$
\begin{aligned}
& s_{0}(q, 0)=-\frac{1}{2}, s_{2}(q, 0)=\frac{1-q^{2}}{12}, \\
& s_{4}(q, 0)=\frac{1-q^{2}}{720}\left(7 q^{2}+17\right) .
\end{aligned}
$$

Now from (2.45) we see that in the absence of the magnetic flux the topological part in the FC vanishes on the string axis.

Combining the expressions (2.32) and (2.41), the total FC is written in the form

$$
\begin{aligned}
\langle\bar{\psi} \psi\rangle= & \langle\bar{\psi} \psi\rangle_{c}^{(0)}-\frac{4 m^{3}}{\pi^{2}} \sum_{l=0}^{\infty} \cos (2 \pi l \tilde{\beta}) \\
& \times\left[\sum_{k=1}^{p}(-1)^{k} \cos (\pi k / q) \cos \left(2 \pi k \alpha_{0}\right) f_{1}\left(m L \sqrt{l^{2}+\rho_{k}^{2}}\right)\right. \\
& \left.+\frac{q}{\pi} \int_{0}^{\infty} \mathrm{d} x \frac{h\left(q, \alpha_{0}, x\right) \sinh x}{\cosh (2 q x)-\cos (q \pi)} f_{1}\left(m L \sqrt{l^{2}+\eta^{2}(x)}\right)\right] .
\end{aligned}
$$

Here also, the prime means that the term with $l=0$ should be taken with the factor $1 / 2$. The latter represents the part $\langle\bar{\psi} \psi\rangle_{s}^{\text {ren }}$. The second term in the right-hand side of this formula encodes the effects from the string and from the flux running along its axis. At large distances from the string, $m r \gg 1$, these effects are suppressed by the factor $e^{-2 m r \sin (\pi / q)}$ for $q>2$ and by the factor $e^{-2 m r}$ for $1 \leqslant q \leqslant 2$, and one has $\langle\bar{\psi} \psi\rangle \approx\langle\bar{\psi} \psi\rangle_{c}^{(0)}$.
In the limit $m L \ll 1$, the asymptotic behavior of the topological part in the FC depends crucially on the parameter $\tilde{\beta}$, if it is zero or not. For $m L \ll 1$, the dominant contribution in the second term of the right-hand side in (2.48) comes from large values of $l$ and we can replace the summation over $l$ by the integration. By using the integration formula from [74], for the corresponding integral we find

$\int_{0}^{\infty} \mathrm{d} l \cos (a l) f_{1}\left(\sqrt{l^{2} c^{2}+b^{2}}\right)=\frac{\pi}{2 b c} e^{-b \sqrt{(a / c)^{2}+1}}$.

For $\tilde{\beta}=0$ this gives

$\langle\bar{\psi} \psi\rangle \approx\langle\bar{\psi} \psi\rangle_{c}^{(0)}+\frac{2}{L}\langle\bar{\psi} \psi\rangle_{s}^{(D=2)}$

where

$$
\begin{aligned}
\langle\bar{\psi} \psi\rangle_{s}^{(D=2)}= & -\frac{m}{2 \pi r}\left[\sum_{k=1}^{p} \frac{(-1)^{k} \cos (\pi k / q)}{s_{k} e^{2 m r s_{k}}} \cos \left(2 \pi k \alpha_{0}\right)\right. \\
& \left.+\frac{q}{\pi} \int_{0}^{\infty} \mathrm{d} x \frac{h\left(q, \alpha_{0}, x\right) \sinh x}{\cosh (2 q x)-\cos (q \pi)} \frac{e^{-2 m r \cosh x}}{\cosh x}\right]
\end{aligned}
$$

is the FC in a $(2+1)$-dimensional conical spacetime $(D=2)$ [67]. An additional coefficient 2 in (2.50) is related to the fact that the number of spinor components in $D=2$ is two instead of four as in the case that $D=3$. For $\tilde{\beta} \neq 0$, in (2.48) the part induced by the string is suppressed by the factor $\exp [-4 \pi \tilde{\beta} r \sin (\pi / q) / L]$ for $q>2$ and by the factor $\exp (-4 \pi \tilde{\beta} r / L)$ for $1 \leqslant q \leqslant 2$.

Figure 2 shows the quantity $\langle\bar{\psi} \psi\rangle / \mathrm{m}^{3}$ as a function of the parameters $\alpha_{0}$ and $\tilde{\beta}$ for $m r=0.25, m L=0.5$ in the geometry of the cosmic string with $q=2.5$. As from the

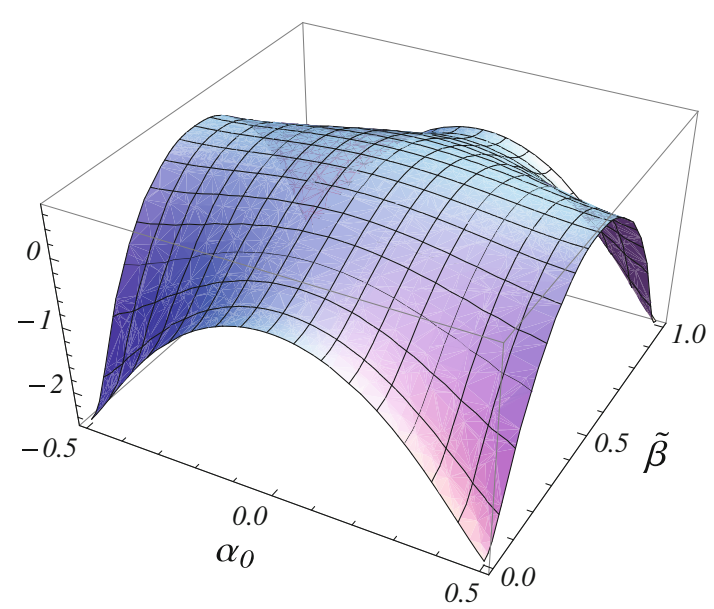

Fig. $2 \mathrm{FC}$ as a function of the parameters $\alpha_{0}$ and $\tilde{\beta}$ for $m r=0.25$, $m L=0.5$ in the geometry of the cosmic string with $q=2.5$ 


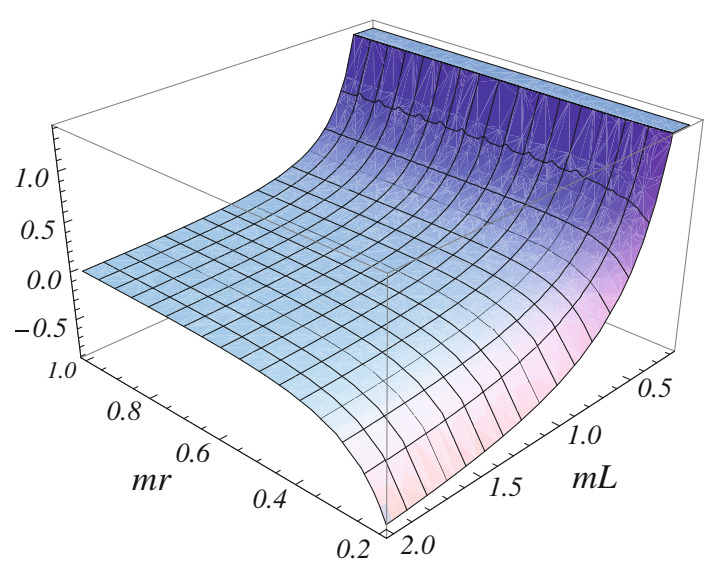

Fig. 3 FC as a function of the distance from the string and of the length of the compactification for $\alpha_{0}=\tilde{\beta}=0.5$ and $q=2.5$

previous analysis, the dependence of $\mathrm{FC}$ on the parameters can provide positive or negative values.

The dependence of the FC on the distance from the string and on the length of the compactification is displayed in Fig. 3 for the special values $\alpha_{0}=\tilde{\beta}=0.5$ and for $q=2.5$.

\section{Energy-momentum tensor}

In this section we analyze another important characteristic of the fermionic vacuum, the VEV of the energy-momentum tensor. For a charged fermionic field, in the presence of an electromagnetic field, the operator of the energy-momentum tensor is expressed as

$T_{\mu \nu}=\frac{i}{2}\left[\bar{\psi} \gamma_{(\mu} \mathcal{D}_{\nu)} \psi-\left(\mathcal{D}_{(\mu} \bar{\psi}\right) \gamma_{\nu)} \psi\right]$,

where $\mathcal{D}_{\mu} \bar{\psi}=\partial_{\mu} \bar{\psi}-i e A_{\mu} \bar{\psi}-\bar{\psi} \Gamma_{\mu}$, and the brackets in the index expression mean the symmetrization over the enclosed indices. Similar to the case of the FC, the VEV of the energymomentum tensor, $\left\langle 0\left|T_{\mu \nu}\right| 0\right\rangle \equiv\left\langle T_{\mu \nu}\right\rangle$, can be evaluated by using the mode-sum formula

$\left\langle T_{\mu \nu}\right\rangle=\frac{i}{2} \sum_{\sigma}\left[\bar{\psi}_{\sigma}^{(-)} \gamma_{(\mu} \mathcal{D}_{\nu)} \psi_{\sigma}^{(-)}-\left(\mathcal{D}_{(\mu} \bar{\psi}_{\sigma}\right) \gamma_{\nu)} \psi_{\sigma}^{(-)}\right]$,

with the notation (2.17). As in the case of the FC, we assume the presence of a cutoff function. We shall evaluate separately all components of the energy-momentum tensor.

In the geometry under consideration, the VEV of the energy-momentum tensor can be decomposed as

$\left\langle T_{\mu}^{v}\right\rangle=\left\langle T_{\mu}^{v}\right\rangle_{s}+\left\langle T_{\mu}^{v}\right\rangle_{c}$,

where the term $\left\langle T_{\mu}^{v}\right\rangle_{s}$ is the VEV for the geometry of an infinite straight cosmic string, and $\left\langle T_{\mu}^{\nu}\right\rangle_{c}$ is the contribu- tion coming from the compactification of the string along its axis. An important point to be mentioned here is that the compactification does not change the local geometry and, hence, the divergences in the VEVs of $\left\langle T_{\mu}^{\nu}\right\rangle$ and $\left\langle T_{\mu}^{\nu}\right\rangle_{s}$ are the same. Thus, by the decomposition (3.3), the renormalization of $\left\langle T_{\mu}^{\nu}\right\rangle$ is reduced to the one for $\left\langle T_{\mu}^{\nu}\right\rangle_{s}$. As in the case of the FC, an explicit decomposition of the form (3.3) can be obtained by using the summation formula (2.21).

\subsection{Energy density}

Let us first consider the energy density, $\left\langle T_{0}^{0}\right\rangle$. By taking into account that $A_{0}$ and $\Gamma_{0}$ vanish and $\partial_{t} \psi_{\sigma}^{(-)}=i E \psi_{\sigma}^{(-)}$, we can see that the contributions of the terms $s=1$ and $s=-1$ are the same. After the summation over $s$ the mode-sum (3.2) for the energy density is reduced to

$\left\langle T_{0}^{0}\right\rangle=-\frac{q}{2 \pi L} \sum_{j} \sum_{l=-\infty}^{+\infty} \int_{0}^{\infty} \mathrm{d} \lambda \lambda E\left[J_{\beta_{j}}^{2}(\lambda r)+J_{\beta_{j}+\epsilon_{j}}^{2}(\lambda r)\right]$.

The summation over the quantum number $l$ can be developed by using the formula (2.21) with $f(k)=\left(k^{2}+\lambda^{2}+m^{2}\right)^{1 / 2}$. The parts in the VEV of the energy density $\left\langle T_{0}^{0}\right\rangle_{s}$ and $\left\langle T_{0}^{0}\right\rangle_{c}$ correspond to the first and second terms in the right-hand side of (2.21), respectively.

We start with the evaluation of $\left\langle T_{0}^{0}\right\rangle_{s}$. It is written in the form

$$
\begin{aligned}
\left\langle T_{0}^{0}\right\rangle_{s}= & -\frac{q}{2 \pi^{2}} \sum_{j} \int_{0}^{\infty} \mathrm{d} \lambda \lambda \int_{0}^{\infty} \mathrm{d} k \sqrt{k^{2}+\lambda^{2}+m^{2}} \\
& \times\left[J_{\beta_{j}}^{2}(\lambda r)+J_{\beta_{j}+\epsilon_{j}}^{2}(\lambda r)\right] .
\end{aligned}
$$

To represent this expression in a form more suitable for the renormalization and for numerical calculations of the renormalized part, we use the relation

$\sqrt{k^{2}+\lambda^{2}+m^{2}}=-\frac{2}{\sqrt{\pi}} \int_{0}^{\infty} d s \partial_{s^{2}} e^{-\left(k^{2}+\lambda^{2}+m^{2}\right) s^{2}}$

After the substitution of (3.6) into (3.5), the integration over $k$ is elementary and the integral over $\lambda$ is evaluated by using (2.25). As a result, the VEV of the energy density is written as

$\left\langle T_{0}^{0}\right\rangle_{s}=-\frac{q}{2 \pi^{2} r^{4}} \int_{0}^{\infty} \mathrm{d} y y^{1 / 2} \partial_{y}\left[y^{3 / 2} e^{-y-m^{2} r^{2} /(2 y)} \mathcal{J}\left(q, \alpha_{0}, y\right)\right]$, 
where the expression for $\mathcal{J}\left(q, \alpha_{0}, y\right)$ is given by (2.28). The first term in the right-hand side of (2.28), $2 e^{y} / q$, provides the divergent part of the VEV. This contribution corresponds to the VEV of the energy density in Minkowski spacetime and in the absence of magnetic fluxes. The well-defined physical result for the energy density is obtained by applying the renormalization procedure, which is reduced to the subtraction of the Minkoswskian counterpart.

So, by making use of (2.28), after the integration over $y$, the renormalized energy density is expressed as

$$
\begin{aligned}
\left\langle T_{0}^{0}\right\rangle_{s}^{\mathrm{ren}}= & \frac{2 m^{4}}{\pi^{2}}\left[\sum_{k=1}^{p}(-1)^{k} \cos (\pi k / q) \cos \left(2 \pi k \alpha_{0}\right) f_{2}\left(2 m r s_{k}\right)\right. \\
& \left.+\frac{q}{\pi} \int_{0}^{\infty} \mathrm{d} x \frac{h\left(q, \alpha_{0}, x\right) \sinh x}{\cosh (2 q x)-\cos (q \pi)} f_{2}(2 m r \cosh x)\right],
\end{aligned}
$$

with the notation (2.33). For the special case defined in (2.30), this expression is reduced to

$$
\left\langle T_{0}^{0}\right\rangle_{s}^{\text {ren }}=\frac{m^{4}}{\pi^{2}} \sum_{k=1}^{q-1} \cos (\pi k / q) \cos ((2 n+1) \pi k / q) f_{2}\left(2 m r s_{k}\right)
$$

Now we turn to the part in the VEV of the energy density induced by the compactification. This part is given by the second integral in the right-hand side of (2.21). By using the expansion (2.37), after the integration over $k$ we can write the topological part in the form

$$
\begin{aligned}
\left\langle T_{0}^{0}\right\rangle_{c}= & \frac{q}{\pi^{2} L} \sum_{l=1}^{\infty} \frac{\cos (2 \pi l \tilde{\beta})}{l} \sum_{j} \int_{0}^{\infty} \mathrm{d} \lambda \lambda \sqrt{\lambda^{2}+m^{2}} \\
& \times K_{1}\left(l L \sqrt{\lambda^{2}+m^{2}}\right)\left[J_{\beta_{j}}^{2}(\lambda r)+J_{\beta_{j}+\epsilon_{j}}^{2}(\lambda r)\right] .
\end{aligned}
$$

By taking into account $K_{1}(x)=-K_{0}^{\prime}(x)$ and using the integral representation (2.39), the integral over $\lambda$ is evaluated with the help of (2.25). This leads to the representation

$$
\begin{aligned}
\left\langle T_{0}^{0}\right\rangle_{c}= & \frac{q}{\pi^{2} r^{2}} \sum_{l=1}^{\infty} \frac{\cos (2 \pi l \tilde{\beta})}{l^{2} L^{2}} \int_{0}^{\infty} \mathrm{d} y e^{-y l^{2} L^{2} /\left(2 r^{2}\right)-r^{2} m^{2} /(2 y)} \\
& \times\left(\partial_{y}+\frac{r^{2} m^{2}}{2 y^{2}}\right) y e^{-y} \mathcal{J}\left(q, \alpha_{0}, y\right)
\end{aligned}
$$

In the part of the integral with $\partial_{y}$ we integrate by parts with the result

$$
\begin{aligned}
\left\langle T_{0}^{0}\right\rangle_{c}= & \frac{q}{2 \pi^{2} r^{4}} \sum_{l=1}^{\infty} \cos (2 \pi l \tilde{\beta}) \\
& \times \int_{0}^{\infty} \mathrm{d} y y e^{-y\left[1+l^{2} L^{2} /\left(2 r^{2}\right)\right]-r^{2} m^{2} /(2 y)} \mathcal{J}\left(q, \alpha_{0}, y\right) .
\end{aligned}
$$

From this it follows that for $\tilde{\beta}=0$ the topological part in the VEV of the energy density is a monotonically decreasing positive function with respect to both $L$ and $m$. Now, by using (2.28), after the integration over $y$ we arrive at the final expression:

$$
\begin{aligned}
\left\langle T_{0}^{0}\right\rangle_{c}= & \frac{4 m^{4}}{\pi^{2}} \sum_{l=1}^{\infty} \cos (2 \pi l \tilde{\beta}) \\
& \times\left[\sum_{k=0}^{p}(-1)^{k} \cos (\pi k / q) \cos \left(2 \pi k \alpha_{0}\right) f_{2}\left(m L \sqrt{l^{2}+\rho_{k}^{2}}\right)\right. \\
& +\frac{q}{\pi} \int_{0}^{\infty} \mathrm{d} x \frac{h\left(q, \alpha_{0}, x\right) \sinh x}{\cosh (2 q x)-\cos (q \pi)} f_{2}\left(m L \sqrt{\left.l^{2}+\eta^{2}(x)\right)}\right],
\end{aligned}
$$

where $\rho_{k}$ and $\eta(z)$ are defined in (2.42). In the special case defined in (2.30), we obtain

$$
\begin{aligned}
\left\langle T_{0}^{0}\right\rangle_{c}= & \frac{2 m^{4}}{\pi^{2}} \sum_{l=1}^{\infty} \cos (2 \pi l \tilde{\beta}) \sum_{k=0}^{q-1} \cos [(2 \pi k / q)(n+1 / 2)] \\
& \times \cos (\pi k / q) f_{2}\left(m L \sqrt{l^{2}+\rho_{k}^{2}}\right)
\end{aligned}
$$

\subsection{Radial stress}

Our next step is the evaluation of the radial stress, $\left\langle T_{r}^{r}\right\rangle$. In order to proceed, we take $A_{r}=\Gamma_{r}=0$ in the general definition of the covariant derivative of the fermionic field. In this way, we can write

$$
\left\langle T_{r}^{r}\right\rangle=\frac{i}{2} \sum_{\sigma}\left[\bar{\psi}_{\sigma}^{(-)} \gamma^{r}\left(\partial_{r} \psi_{\sigma}^{(-)}\right)-\left(\partial_{r} \bar{\psi}_{\sigma}^{(-)}\right) \gamma^{r} \psi_{\sigma}^{(-)}\right] .
$$

Substituting the mode functions from (2.8) into the above expression, after some intermediate steps, we arrive at

$$
\begin{aligned}
\left\langle T_{r}^{r}\right\rangle= & -\frac{q}{4 \pi L} \sum_{\sigma} \frac{\epsilon_{j} \lambda^{3}}{E}\left[J_{\beta_{j}}^{\prime}(\lambda r) J_{\beta_{j}+\epsilon_{j}}(\lambda r)\right. \\
& \left.-J_{\beta_{j}}(\lambda r) J_{\beta_{j}+\epsilon_{j}}^{\prime}(\lambda r)\right],
\end{aligned}
$$

where the prime means the derivative with respect to the argument of the function. By using the recurrent relations for the Bessel functions, after the summation over $s$, we write the VEV in the form 
$\left\langle T_{r}^{r}\right\rangle=\frac{q}{2 \pi L} \sum_{l=-\infty}^{+\infty} \int_{0}^{\infty} \mathrm{d} \lambda \frac{\lambda^{3}}{E} S(\lambda r)$,

with the function

$S(x)=\sum_{j}\left[J_{\beta_{j}}^{2}(x)+J_{\beta_{j}+\epsilon_{j}}^{2}(x)-\frac{2 \beta_{j}+\epsilon_{j}}{x} J_{\beta_{j}}(x) J_{\beta_{j}+\epsilon_{j}}(x)\right]$.

After the application of the summation formula (2.21) to the series over $l$ in (3.17), the radial stress is decomposed into the parts $\left\langle T_{r}^{r}\right\rangle_{s}$ and $\left\langle T_{r}^{r}\right\rangle_{c}$.

We start with the calculation of the part corresponding to the geometry of a straight cosmic string:

$$
\left\langle T_{r}^{r}\right\rangle_{s}=\frac{2 q}{(2 \pi)^{2}} \int_{0}^{\infty} \mathrm{d} \lambda \lambda^{3} \int_{0}^{\infty} \mathrm{d} k \frac{S(\lambda r)}{\sqrt{k^{2}+\lambda^{2}+m^{2}}}
$$

Using the relation (2.24) we can develop the integration over $k$. After that we can write the above expression in the form

$$
\left\langle T_{r}^{r}\right\rangle_{s}=\frac{2 q}{(2 \pi)^{2}} \int_{0}^{\infty} \mathrm{d} s \frac{e^{-m^{2} s^{2}}}{s} \int_{0}^{\infty} \mathrm{d} \lambda \lambda^{3} e^{-\lambda^{2} s^{2}} S(\lambda r) .
$$

Compared with the case of the energy density, the integral over $\lambda$ is more delicate. Below we present the main steps:

$$
\begin{aligned}
& \int_{0}^{\infty} \mathrm{d} \lambda \lambda^{3} e^{-\lambda^{2} s^{2}} S(\lambda r)=\sum_{j} \int_{0}^{\infty} \mathrm{d} \lambda \lambda^{3} e^{-\lambda^{2} s^{2}} \\
& \quad \times\left[J_{\beta_{j}}^{2}(\lambda r)+J_{\beta_{j}+\epsilon_{j}}^{2}(\lambda r)\right] \\
& \quad-\sum_{j} \frac{2 \beta_{j}+\epsilon_{j}}{r} \int_{0}^{\infty} \mathrm{d} \lambda \lambda^{2} e^{-\lambda^{2} s^{2}} J_{\beta_{j}}(\lambda r) J_{\beta_{j}+\epsilon_{j}}(\lambda r) .
\end{aligned}
$$

The first integral on the right-hand side of (3.21) can be developed taking the derivative $-\partial_{S^{2}}$ on the integral in the left-hand side of (2.25). As to the second integral, by using the relation $J_{\beta_{j}}(\lambda r) J_{\beta_{j}+\epsilon_{j}}(\lambda r)=\frac{1}{2 \lambda}\left(-\epsilon_{j} \partial_{r}+2 \beta_{j} / r\right) J_{\beta_{j}}^{2}(\lambda r)$,

we can show that

$$
\begin{gathered}
\int_{0}^{\infty} \mathrm{d} \lambda \lambda^{2} e^{-\lambda^{2} s^{2}} J_{\beta_{j}}(\lambda r) J_{\beta_{j}+\epsilon_{j}}(\lambda r) \\
\quad=\frac{r \epsilon_{j} e^{-y}}{4 s^{4}}\left[I_{\beta_{j}}(y)-I_{\beta_{j}+\epsilon_{j}}(y)\right],
\end{gathered}
$$

with $y=r^{2} /\left(2 s^{2}\right)$. Further, we use the relation

$$
\begin{aligned}
& \left(1+2 \epsilon_{j} \beta_{j}\right)\left[I_{\beta_{j}}(y)-I_{\beta_{j}+\epsilon_{j}}(y)\right] \\
& \quad=2\left(y \partial_{y}-y+1 / 2\right)\left[I_{\beta_{j}}(y)+I_{\beta_{j}+\epsilon_{j}}(y]\right.
\end{aligned}
$$

to express the term on the right-hand side of (3.22) as the sum of the modified Bessel functions. Combining all these results, for the VEV of the radial stress we obtain

$\left.\left\langle T_{r}^{r}\right\rangle_{s}=\frac{q}{4 \pi^{2} r^{4}} \int_{0}^{\infty} \mathrm{d} y y e^{-y-m^{2} r^{2} /(2 y)} \mathcal{J}\left(q, \alpha_{0}, y\right)\right]$.

By the direct substitution of (2.28) into (3.24), we can see that the divergent part coming from the first term in the righthand side of (2.28) represents the corresponding quantity on the topologically trivial Minkowski spacetime in the absence of fluxes. Applying again the standard renormalization procedure, we see that the renormalized expression for the radial stress has the same structure as that obtained from (3.7) after integration by parts. Consequently, we conclude that

$$
\left\langle T_{r}^{r}\right\rangle_{s}^{\mathrm{ren}}=\left\langle T_{0}^{0}\right\rangle_{s}^{\mathrm{ren}}
$$

Now we pass to the evaluation of the part $\left\langle T_{r}^{r}\right\rangle_{c}$. This contribution comes from the second integral on the right-hand side of the summation formula (2.21). By using the expansion (2.37), the integral over $k$ is expressed in terms of the function $K_{0}\left(l L \sqrt{m^{2}+\lambda^{2}}\right)$. With the help of the integral representation (2.39) for the Macdonald function, the expression for the topological part becomes

$$
\begin{aligned}
\left\langle T_{r}^{r}\right\rangle_{c}= & \frac{q}{2 \pi^{2}} \sum_{l=1}^{\infty} \cos (2 \pi l \tilde{\beta}) \int_{0}^{\infty} \mathrm{d} t \frac{e^{-t-l^{2} L^{2} m^{2} /(4 t)}}{t} \\
& \times \int_{0}^{\infty} \mathrm{d} \lambda \lambda^{3} e^{-l^{2} L^{2} \lambda^{2} /(4 t)} S(\lambda r) .
\end{aligned}
$$

The integral over $\lambda$ is in the form (3.21). So the result is

$$
\begin{aligned}
& \int_{0}^{\infty} \mathrm{d} \lambda \lambda^{3} e^{-l^{2} L^{2} \lambda^{2} /(4 t)} S(\lambda r) \\
& \quad=\frac{y^{2}}{r^{4}} e^{-y} \sum_{j}\left[I_{\beta_{j}}(y)+I_{\beta_{j}+\epsilon_{j}}(y)\right],
\end{aligned}
$$

with the notation $y=2 r^{2} t /\left(l^{2} L^{2}\right)$. Finally we arrive at the expression which coincides with (3.12). Hence, for the topological parts as well we have the relation

$$
\left\langle T_{r}^{r}\right\rangle_{c}=\left\langle T_{0}^{0}\right\rangle_{c}
$$


Note that, for a scalar field with general curvature coupling parameter, the VEVs of the radial stress and the energy density are different [18].

\subsection{Azimuthal stress}

In the evaluation of the VEV for the azimuthal stress, $\left\langle T_{\phi}^{\phi}\right\rangle$, we have to take into account, $A_{\phi}=q \alpha / e$ and

$$
\begin{aligned}
\Gamma_{\phi}=\frac{1-q}{2} \gamma^{(1)} \gamma^{(2)} & =-\frac{i}{2}(1-q) \Sigma^{(3)}, \\
\Sigma^{(3)} & =\operatorname{diag}\left(\sigma_{3}, \sigma_{3}\right),
\end{aligned}
$$

$\sigma_{3}$ being a Pauli matrix. Thus, this component reads

$\left\langle T_{\phi}^{\phi}\right\rangle=\frac{i}{2} \sum_{\sigma}\left[\bar{\psi}_{\sigma}^{(-)} \gamma^{\phi} \mathcal{D}_{\phi} \psi_{\sigma}^{(-)}-\left(\mathcal{D}_{\phi} \bar{\psi}_{\sigma}^{(-)}\right) \gamma^{\phi} \psi_{\sigma}^{(-)}\right]$.

In the development of the term inside the bracket, it is convenient to express the angular derivative in terms of the total angular momentum operator: $\partial_{\phi}=i \widehat{J_{3}}-i(q / 2) \Sigma^{(3)}$. Moreover, we can observe that the anticommutator, $\left\{\gamma^{\phi}, \Sigma^{(3)}\right\}$, which appears in the development, vanishes. Therefore, after some steps, we get

$\left\langle T_{\phi}^{\phi}\right\rangle=q \sum_{\sigma}(j+\alpha) \bar{\psi}_{\sigma}^{(-)} \gamma^{\phi} \psi_{\sigma}^{(-)}$.

Now substituting the Dirac matrix $\gamma^{\phi}$ and the expression for the negative-energy wave function, we obtain

$$
\left\langle T_{\phi}^{\phi}\right\rangle=\frac{q^{2}}{2 \pi L r} \sum_{\sigma} \epsilon_{j}(j+\alpha) \frac{\lambda^{2}}{E} J_{\beta_{j}}(\lambda r) J_{\beta_{j}+\epsilon_{j}}(\lambda r) .
$$

The summation over $s$ provides the factor 2 and for the summation over $l$ we use again (2.21). In this way, we have for the azimuthal stress the decomposition (3.3).

Let us start with the part corresponding to the straight cosmic string:

$$
\begin{aligned}
\left\langle T_{\phi}^{\phi}\right\rangle_{s}= & \frac{q^{2}}{\pi^{2} r} \sum_{j} \epsilon_{j}(j+\alpha) \int_{0}^{\infty} \mathrm{d} \lambda \lambda^{2} \\
& \times \int_{0}^{\infty} \mathrm{d} k \frac{J_{\beta_{j}}(\lambda r) J_{\beta_{j}+\epsilon_{j}}(\lambda r)}{\sqrt{k^{2}+\lambda^{2}+m^{2}}} .
\end{aligned}
$$

Using again the relation (2.24), we can develop the integral over $k$. For the integral over $\lambda$ we use (3.22). Finally defining the new variable $y=r^{2} /\left(2 s^{2}\right)$ we get

$$
\begin{aligned}
\left\langle T_{\phi}^{\phi}\right\rangle_{s}= & \frac{q^{2}}{2 \pi^{2} r^{4}} \sum_{j}(j+\alpha) \\
& \times \int_{0}^{\infty} \mathrm{d} y y e^{-y-m^{2} r^{2} /(2 y)}\left[I_{\beta_{j}}(y)-I_{\beta_{j}+\epsilon_{j}}(y)\right] .(3
\end{aligned}
$$

By taking into account that $q(j+\alpha)=\epsilon_{j} \beta_{j}+1 / 2$ and using the relation (3.23), we represent (3.34) in the form

$$
\begin{aligned}
\left\langle T_{\phi}^{\phi}\right\rangle_{s}= & \frac{q}{2 \pi^{2} r^{4}} \\
& \left.\times \int_{0}^{\infty} \mathrm{d} y y e^{-m^{2} r^{2} /(2 y)}\left(y \partial_{y}+1 / 2\right) e^{-y} \mathcal{J}\left(q, \alpha_{0}, y\right)\right] .
\end{aligned}
$$

Comparing this expression with (3.24) one can see that the following relation holds:

$\left\langle T_{\phi}^{\phi}\right\rangle_{s}=\left(r \partial_{r}+1\right)\left\langle T_{r}^{r}\right\rangle_{s}$

Combining (3.36) with the expression for $\left\langle T_{r}^{r}\right\rangle_{s}$ and by using the relations

$f_{v}^{\prime}(x)=-x f_{v+1}(x), x^{2} f_{v+1}(x)=2 v f_{v}(x)+f_{v-1}(x)$,

for the renormalized VEV of the azimuthal stress we find the following result:

$$
\begin{aligned}
\left\langle T_{\phi}^{\phi}\right\rangle_{s}^{\mathrm{ren}}= & \frac{2 m^{4}}{\pi^{2}}\left[\sum_{k=1}^{p}(-1)^{k} \cos (\pi k / q) \cos \left(2 \pi k \alpha_{0}\right) F_{\phi}^{(0)}\left(2 m r s_{k}\right)\right. \\
& \left.+\frac{q}{\pi} \int_{0}^{\infty} \mathrm{d} x \frac{h\left(q, \alpha_{0}, x\right) \sinh x}{\cosh (2 q x)-\cos (q \pi)} F_{\phi}^{(0)}(2 m r \cosh x)\right],
\end{aligned}
$$

with the notation

$F_{\phi}^{(0)}(x)=\partial_{x}\left[x f_{2}(x)\right]=f_{2}(x)-x^{2} f_{3}(x)=-f_{1}(x)-3 f_{2}(x)$.

Now we start the evaluation of the contribution to the azimuthal stress due to the compactification. This term is given by the substitution of the second integral in (2.21) into (3.32). By using the expansion (2.37), the integral over $k$ provides us with $K_{0}\left(l L \sqrt{m^{2}+\lambda^{2}}\right)$. On the base of the integral representation (2.39) we can rewrite the above expression as 


$$
\begin{aligned}
\left\langle T_{\phi}^{\phi}\right\rangle_{c}= & \frac{q^{2}}{\pi^{2} r} \sum_{l=1}^{\infty} \cos (2 \pi l \tilde{\beta}) \int_{0}^{\infty} \mathrm{d} t \frac{e^{-t-l^{2} L^{2} m^{2} /(4 t)}}{t} \\
& \times \sum_{j} \epsilon_{j}(j+\alpha) \\
& \times \int_{0}^{\infty} \mathrm{d} \lambda \lambda^{2} e^{-l^{2} L^{2} \lambda^{2} /(4 t)} J_{\beta_{j}}(\lambda r) J_{\beta_{j}+\epsilon_{j}}(\lambda r) .
\end{aligned}
$$

The integral over $\lambda$ can be obtained by using the previous result (3.22). Defining a new variable $y=2 r^{2} t /\left(l^{2} L^{2}\right)$, the expression obtained is given in terms of $q(j+\alpha)\left[I_{\beta_{j}}(y)-\right.$ $\left.I_{\beta_{j}+\epsilon_{j}}(y)\right]$. To continue the development, we use again (3.23) and get

$$
\begin{aligned}
\left\langle T_{\phi}^{\phi}\right\rangle_{c}= & \frac{q}{\pi^{2} r^{4}} \sum_{l=1}^{\infty} \cos (2 \pi l \tilde{\beta}) \\
& \times \int_{0}^{\infty} \mathrm{d} y y e^{-y\left[1+l^{2} L^{2} /\left(2 r^{2}\right)\right]-m^{2} r^{2} /(2 y)} \\
& \left.\times\left(y \partial_{y}-y+1 / 2\right) \mathcal{J}\left(q, \alpha_{0}, y\right)\right] .
\end{aligned}
$$

By using the same trick as for the case of $\left\langle T_{\phi}^{\phi}\right\rangle_{s}$, we can see that from (3.41) the following relation is obtained:

$\left\langle T_{\phi}^{\phi}\right\rangle_{c}=\left(r \partial_{r}+1\right)\left\langle T_{r}^{r}\right\rangle_{c}$

Now combining this with the expression for $\left\langle T_{r}^{r}\right\rangle_{c}$, one gets the final expression for the topological part in the azimuthal stress:

$$
\begin{aligned}
\left\langle T_{\phi}^{\phi}\right\rangle_{c}= & \frac{4 m^{4}}{\pi^{2}} \sum_{l=1}^{\infty} \cos (2 \pi l \tilde{\beta}) \\
& \times\left[\sum_{k=0}^{p}(-1)^{k} \cos (\pi k / q) \cos \left(2 \pi k \alpha_{0}\right) F_{\phi}^{(l)}\left(m L \sqrt{l^{2}+\rho_{k}^{2}}\right)\right. \\
& \left.+\frac{q}{\pi} \int_{0}^{\infty} \mathrm{d} x \frac{h\left(q, \alpha_{0}, x\right) \sinh x}{\cosh (2 q x)-\cos (q \pi)} F_{\phi}^{(l)}\left(m L \sqrt{l^{2}+\eta^{2}(x)}\right)\right],
\end{aligned}
$$

with the function

$F_{\phi}^{(l)}(x)=\left(m^{2} L^{2} l^{2}-x^{2}\right) f_{3}(x)+f_{2}(x)$

Note that for $l=0$ this function coincides with (3.39).

\subsection{Axial stress}

In the calculation of the axial stress, we have to consider $A_{z}=-\Phi_{z} / L$ in the covariant derivative of the field operator. Thus, we have $\mathcal{D}_{z} \psi_{\sigma}^{(-)}=i \tilde{k}_{l} \psi_{\sigma}^{(-)}$. In addition, the matrix $\gamma^{z}$ coincides with the standard expression for the Dirac matrix in flat spacetime. For this component, we have

$$
\left\langle T_{z}^{z}\right\rangle=-\sum_{\sigma} \tilde{k}_{l} \bar{\psi}_{\sigma}^{(-)} \gamma^{z} \psi_{\sigma}^{(-)}
$$

Substituting the expression for the negative-energy mode function into the above expression, one obtains

$$
\begin{aligned}
\left\langle T_{z}^{z}\right\rangle= & -\frac{q}{2 \pi L} \sum_{j} \int_{0}^{\infty} \mathrm{d} \lambda \lambda \\
& \times \sum_{l=-\infty}^{+\infty} \frac{\tilde{k}_{l}^{2}}{E}\left[J_{\beta_{j}}^{2}(\lambda r)+J_{\beta_{j}+\epsilon_{j}}^{2}(\lambda r)\right] .
\end{aligned}
$$

For the summation over $l$, we use the Abel-Plana summation formula, Eq. ( 2.21), taking $f(k)=k^{2}\left(k^{2}+\lambda^{2}+\right.$ $\left.m^{2}\right)^{-1 / 2}$. This allows us to decompose the axial stress in accordance with (3.3). The contribution to the axial stress corresponding to the geometry of a straight cosmic string is given by the first term on the right-hand side of (2.21):

$\left\langle T_{z}^{z}\right\rangle_{s}=\frac{q}{2 \pi^{2}} \sum_{j} \int_{0}^{\infty} \mathrm{d} \lambda \lambda \int_{0}^{\infty} \mathrm{d} k k^{2} \frac{J_{\beta_{j}}^{2}(\lambda r)+J_{\beta_{j}+\epsilon_{j}}^{2}(\lambda r)}{\sqrt{m^{2}+\lambda^{2}+k^{2}}}$.

Using the relation (2.24), the evaluation of the integral over $k$ can be promptly obtained. For the integral over $\lambda$ we use again the result (2.25). Finally, we get

$$
\left.\left\langle T_{z}^{z}\right\rangle_{s}=\frac{q}{4 \pi^{2} r^{4}} \int_{0}^{\infty} \mathrm{d} y y e^{-y-m^{2} r^{2} /(2 y)} \mathcal{J}\left(q, \alpha_{0}, y\right)\right] .
$$

This result coincides with (3.24); consequently we conclude that

$\left\langle T_{z}^{z}\right\rangle_{s}^{\text {ren }}=\left\langle T_{0}^{0}\right\rangle_{s}^{\text {ren }}$

Also, this property directly follows from the invariance of the problem with respect to a boost along the axis of the string.

The topological part in the axial stress is given by substituting the second integral on the right-hand side of (2.21) into (3.46):

$$
\begin{aligned}
\left\langle T_{z}^{z}\right\rangle_{c}= & -\frac{q}{\pi^{2}} \sum_{l=1}^{\infty} \cos (2 \pi l \tilde{\beta}) \int_{0}^{\infty} \mathrm{d} \lambda \lambda \int_{\sqrt{\lambda^{2}+m^{2}}}^{\infty} \mathrm{d} k \\
& \times \frac{k^{2} e^{-l L k}}{\sqrt{k^{2}-\lambda^{2}-m^{2}}} \sum_{j}\left[J_{\beta_{j}}^{2}(\lambda r)+J_{\beta_{j}+\epsilon_{j}}^{2}(\lambda r)\right] .
\end{aligned}
$$


Writing $k^{2} e^{-l L k}=l^{-2} \partial_{L}^{2} e^{-l L k}$ and changing the order of the differentiation and the integration over $k$, the integral is expressed in terms of the function $K_{0}\left(l L \sqrt{m^{2}+\lambda^{2}}\right)$. By using the integral representation (2.39) for the latter we get

$$
\begin{aligned}
\left\langle T_{z}^{z}\right\rangle_{c}= & -\frac{q}{2 \pi^{2} r^{2}} \partial_{L}^{2} \sum_{l=1}^{\infty} \frac{\cos (2 \pi l \tilde{\beta})}{l^{2}} \\
& \times \int_{0}^{\infty} \mathrm{d} y e^{-y\left[1+l^{2} L^{2} /\left(2 r^{2}\right)\right]-m^{2} r^{2} /(2 y)} \mathcal{J}\left(q, \alpha_{0}, y\right) .
\end{aligned}
$$

After differentiation one obtains

$$
\begin{aligned}
\left\langle T_{z}^{z}\right\rangle_{c}= & \frac{q}{2 \pi^{2} r^{4}} \sum_{l=1}^{\infty} \cos (2 \pi l \tilde{\beta}) \int_{0}^{\infty} \mathrm{d} y y\left(1-y l^{2} L^{2} / r^{2}\right) \\
& \times e^{-y\left(1+l^{2} L^{2} /\left(2 r^{2}\right)\right)-m^{2} r^{2} /(2 y)} \mathcal{J}\left(q, \alpha_{0}, y\right) .
\end{aligned}
$$

By using the representation (2.28), after the integration over $y$, we arrive at the final expression

$$
\begin{aligned}
\left\langle T_{z}^{z}\right\rangle_{c}= & \frac{4 m^{4}}{\pi^{2}} \sum_{l=1}^{\infty} \cos (2 \pi l \tilde{\beta}) \\
& \times\left[\sum_{k=0}^{p}(-1)^{k} \cos (\pi k / q) \cos \left(2 \pi k \alpha_{0}\right) F_{z}^{(l)}\left(m L \sqrt{l^{2}+\rho_{k}^{2}}\right)\right. \\
& +\frac{q}{\pi} \int_{0}^{\infty} \mathrm{d} x \frac{h\left(q, \alpha_{0}, x\right) \sinh x}{\cosh (2 q x)-\cos (q \pi)} F_{z}^{(l)}\left(m L \sqrt{\left.l^{2}+\eta^{2}(x)\right)}\right],
\end{aligned}
$$

with the notation

$F_{z}^{(l)}(x)=f_{2}(x)-l^{2} L^{2} m^{2} f_{3}(x)$.

Note that the relation $\left\langle T_{z}^{z}\right\rangle_{c}^{\text {ren }}=\left\langle T_{0}^{0}\right\rangle_{c}^{\text {ren }}$ takes place only for a massless field.

\section{Properties of the energy-momentum tensor and the vacuum energy}

In this section we investigate the properties and the asymptotic behavior of the VEVs found in the previous section. We can check that both contributions to the VEV of the energymomentum tensor obey the trace relation:

$$
\left\langle T_{\mu}^{\mu}\right\rangle_{s}^{\text {ren }}=m\langle\bar{\psi} \psi\rangle_{s}^{\text {ren }},\left\langle T_{\mu}^{\mu}\right\rangle_{c}=m\langle\bar{\psi} \psi\rangle_{c} .
$$

In particular, for a massless field the vacuum energymomentum tensor is traceless. Because the cosmic string spacetime is locally flat for $r>0$, the trace anomaly is zero. Another important property obeyed by the VEV of the energy-momentum tensor is its covariant conservation: $\nabla_{\mu}\left\langle T_{\nu}^{\mu}\right\rangle=0$. For the problem under consideration this equation is reduced to a single differential equation, $\partial_{r}\left(r\left\langle T_{r}^{r}\right\rangle\right)=\left\langle T_{\phi}^{\phi}\right\rangle$. We have already proved this relation for a separate straight cosmic string and for the topological parts during the calculation of the corresponding VEVs in the previous section (see (3.36) and (3.42)).

Combining the formulas obtained above, the part in the VEV of the energy-momentum tensor corresponding to the geometry of a straight cosmic string is represented by (no summation over $\mu$ )

$$
\begin{aligned}
\left\langle T_{\mu}^{\mu}\right\rangle_{s}^{\mathrm{ren}}= & \frac{2 m^{4}}{\pi^{2}}\left[\sum_{k=1}^{p}(-1)^{k} \cos (\pi k / q) \cos \left(2 \pi k \alpha_{0}\right) F_{\mu}^{(0)}\left(2 m r s_{k}\right)\right. \\
& \left.+\frac{q}{\pi} \int_{0}^{\infty} \mathrm{d} x \frac{h\left(q, \alpha_{0}, x\right) \sinh x}{\cosh (2 q x)-\cos (q \pi)} F_{\mu}^{(0)}(2 m r \cosh x)\right],
\end{aligned}
$$

where $F_{\mu}^{(0)}(x)=f_{2}(x)$ for $\mu=0, r, z$, and the function $F_{\phi}^{(0)}(x)$ is defined by the relation (3.39). In the absence of magnetic flux along the string axis one has $\alpha_{0}=0$, and (4.2) is reduced to the expression given in [71]. In this special case an alternative integral representation for $\left\langle T_{\mu}^{\mu}\right\rangle_{s}^{\text {ren }}$ is derived in [63]. The case $q<2, \alpha_{0}=0$ was considered in [46]. For a massless field and for $\alpha_{0}=0$, the renormalized VEV for the energy-momentum tensor was found in [30-32].

For the special case (2.30) with an integer $q$, the integral term in (4.2) vanishes and we get the simple expression

$$
\left\langle T_{\mu}^{\mu}\right\rangle_{s}^{\mathrm{ren}}=\frac{m^{4}}{\pi^{2}} \sum_{k=1}^{q-1} \cos (\pi k / q) \cos ((2 n+1) \pi k / q) F_{\mu}^{(0)}\left(2 m r s_{k}\right) .
$$

Another simplification is possible for a massless field. In this case, by using (2.35), from (4.2) we find (no summation over н)

$\left\langle T_{\mu}^{\mu}\right\rangle_{s}^{\text {ren }}=\delta_{\mu} \frac{s_{4}\left(q, \alpha_{0}\right)}{4 \pi^{2} r^{4}}$,

where

$\delta_{\mu}=1$ for $\mu=0, r, z, \delta_{\phi}=-3$,

and the function $s_{n}\left(q, \alpha_{0}\right)$ is defined in (2.46). Note that, by taking into account (2.47), for $\alpha_{0}=0$, Eq. (4.4) is reduced to the result given in [30-32]. For a massive field, the expression in the right-hand side of (4.4) gives the leading term in the asymptotic expansion of $\left\langle T_{0}^{0}\right\rangle_{s}^{\text {ren }}$ for points near the string, $m r \ll 1$.

At large distance from the string, for $q>2$ the dominant contribution in (4.2) comes from the term $k=1$ and, in the leading order, we find 


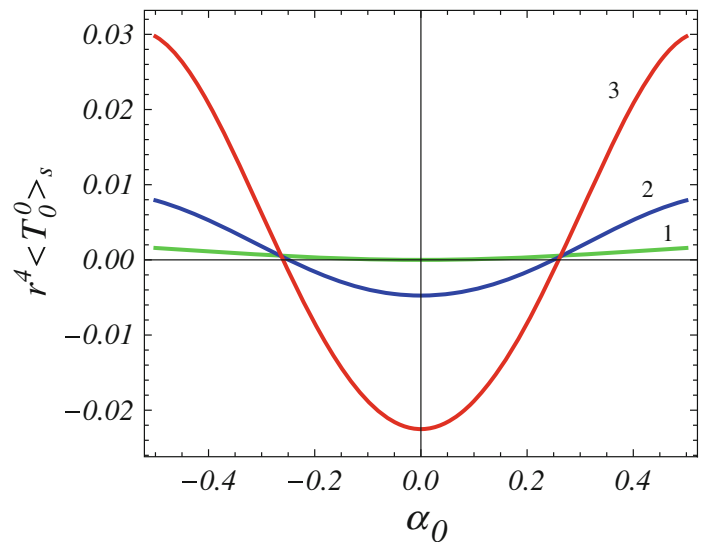

Fig. 4 Quantity $r^{4}\left\langle T_{0}^{0}\right\rangle_{s}^{\text {ren }}$ for a massless fermionic field as a function of $\alpha_{0}$ for different values of $q$ (numbers near the curves)

$\left\langle T_{0}^{0}\right\rangle_{s}^{\mathrm{ren}} \approx-\frac{m^{4} e^{-2 m r \sin (\pi / q)}}{4 \pi^{3 / 2}} \frac{\cos (\pi / q) \cos \left(2 \pi \alpha_{0}\right)}{[m r \sin (\pi / q)]^{5 / 2}}$,

$\left\langle T_{\phi}^{\phi}\right\rangle_{s}^{\text {ren }} \approx-2 m r \sin (\pi / q)\left\langle T_{0}^{0}\right\rangle_{s}^{\text {ren }}$.

For $1 \leqslant q \leqslant 2$ the sum over $k$ in the right-hand side of (4.2) is absent and the integral term is suppressed by the factor $e^{-2 m r}$. In both cases $\left|\left\langle T_{0}^{0}\right\rangle_{s}^{\text {ren }}\right| /\left|\left\langle T_{\phi}^{\phi}\right\rangle_{s}^{\text {ren }}\right| \sim(m r)^{-1} \ll 1$.

In Fig. 4 we have plotted the energy density for a massless fermionic field, multiplied by $r^{4}$, as a function of the parameters $\alpha_{0}$ for different values of $q$ (numbers near the curves). As is seen, with dependence of the magnetic flux, the vacuum energy density can be either positive or negative. In particular, the energy density is negative in the absence of the magnetic flux. The maximal (positive) value of the energy density is obtained for $\Phi_{\phi}=\Phi_{0} / 2$. For some values of $\alpha_{0}$ the VEV of the energy-momentum tensor vanishes. For these values, the effects induced by the topology of the cosmic string spacetime and by the magnetic flux compensate each other.

Now we turn to the investigation of the topological parts in the VEVs for the components of the energy-momentum tensor. The $k=0$ terms in these parts represent the VEVs in Minkowski spacetime with spatial topology $R^{2} \times S^{1}$ in the absence of the cosmic string and of flux along the $z$-axis $\left(q=1, \alpha_{0}=0\right)$. Denoting the corresponding VEV by $\left\langle T_{\mu}^{\mu}\right\rangle_{c}^{(0)}$, from the formulas given above we have (no summation over $\mu$ )

$\left\langle T_{\mu}^{\mu}\right\rangle_{c}^{(0)}=\frac{2 m^{4}}{\pi^{2}} \sum_{l=1}^{\infty} \cos (2 \pi l \tilde{\beta}) F_{\mu}^{(l)}(m L l)$,

with the functions

$F_{0}^{(l)}(x)=F_{r}^{(l)}(x)=f_{2}(x)$,

$F_{\phi}^{(l)}(x)=\left(m^{2} L^{2} l^{2}-x^{2}\right) f_{3}(x)+f_{2}(x)$,

$F_{z}^{(l)}(x)=f_{2}(x)-l^{2} L^{2} m^{2} f_{3}(x)$.
The corresponding results for a more general topology $R^{p} \times\left(S^{1}\right)^{D-p}$ are given in [15]. Now, combining the formulas obtained before for separate components of the energymomentum tensor, we can see that the total VEV is written in the form (no summation over $\mu$ )

$$
\begin{aligned}
\left\langle T_{\mu}^{\mu}\right\rangle= & \left\langle T_{\mu}^{\mu}\right\rangle_{c}^{(0)}+\frac{4 m^{4}}{\pi^{2}} \sum_{l=0}^{\infty} \cos (2 \pi l \tilde{\beta}) \\
& \times\left[\sum_{k=1}^{p}(-1)^{k} \cos (\pi k / q) \cos \left(2 \pi k \alpha_{0}\right) F_{\mu}^{(l)}\left(m L \sqrt{l^{2}+\rho_{k}^{2}}\right)\right. \\
& +\frac{q}{\pi} \int_{0}^{\infty} \mathrm{d} x \frac{h\left(q, \alpha_{0}, x\right) \sinh x}{\cosh (2 q x)-\cos (q \pi)} F_{\mu}^{(l)}\left(m L \sqrt{\left.l^{2}+\eta^{2}(x)\right)}\right],
\end{aligned}
$$

where the prime on the sum over $l$, as before, means that the term with $l=0$ should be taken with the coefficient $1 / 2$. The latter coincides with $\left\langle T_{\mu}^{\mu}\right\rangle_{s}$ and the $l \neq 0$ terms come from the compactification of the string along its axis. The VEV (4.9) is an even periodic function of $\tilde{\beta}$ and $\alpha_{0}$ with period equal to 1. It is symmetric under the replacement $\tilde{\beta} \rightarrow 1-\tilde{\beta}$.

Simpler expressions for the VEVs are obtained in two particular cases. For integer values of the parameter $q$ and for $\alpha_{0}$ given by (2.30) one has $h\left(q, \alpha_{0}, x\right)=0$ and (4.9) is reduced to

$$
\begin{aligned}
\left\langle T_{\mu}^{\mu}\right\rangle= & \left\langle T_{\mu}^{\mu}\right\rangle_{c}^{(0)}+\frac{2 m^{4}}{\pi^{2}} \sum_{k=1}^{q-1} \cos (\pi k / q) \cos ((2 n+1) \pi k / q) \\
& \times \sum_{l=0}^{\infty} \cos (2 \pi l \tilde{\beta}) F_{\mu}^{(l)}\left(m L \sqrt{l^{2}+\rho_{k}^{2}}\right)
\end{aligned}
$$

For a massless field, by using the asymptotic expression (2.35), one gets (no summation over $\mu$ )

$$
\begin{aligned}
\left\langle T_{\mu}^{\mu}\right\rangle= & \left\langle T_{\mu}^{\mu}\right\rangle_{c}^{(0)}+\frac{8 \delta_{\mu}}{\pi^{2} L^{4}} \\
& \times\left[\sum_{k=1}^{p}(-1)^{k} \cos (\pi k / q) \cos \left(2 \pi k \alpha_{0}\right) C\left(\tilde{\beta}, \rho_{k}\right)\right. \\
& \left.+\frac{q}{\pi} \int_{0}^{\infty} \mathrm{d} x \frac{h\left(q, \alpha_{0}, x\right) C(\tilde{\beta}, \eta(x)) \sinh x}{\cosh (2 q x)-\cos (q \pi)}\right]
\end{aligned}
$$

where $\delta_{\mu}$ is given by (4.5) and we have defined the function

$C(\tilde{\beta}, x)=\sum_{l=0}^{\infty} \frac{\cos (2 \pi l \tilde{\beta})}{\left(l^{2}+x^{2}\right)^{2}}$.

For the series (4.12) one has [75]

$$
\begin{aligned}
C(\tilde{\beta}, x)= & \frac{\pi^{2} \cosh (2 \pi \tilde{\beta} x)}{4 x^{2} \sinh ^{2}(\pi x)} \\
& +\pi \frac{\cosh [\pi(1-2 \tilde{\beta}) x]+2 \pi \tilde{\beta} x \sinh [\pi(1-2 \tilde{\beta}) x]}{4 x^{3} \sinh (\pi x)},
\end{aligned}
$$


with $0 \leqslant \tilde{\beta} \leqslant 1$. In this case, the Minkowskian part reads

$$
\begin{aligned}
\left\langle T_{\mu}^{\mu}\right\rangle_{c}^{(0)} & =\frac{4 \delta_{\mu}}{\pi^{2} L^{4}} \lim _{x \rightarrow 0}\left[C(\tilde{\beta}, x)-\frac{1}{2 x^{4}}\right] \\
& =\frac{4 \pi^{2} \delta_{\mu}}{3 L^{4}}\left[\frac{1}{30}-\tilde{\beta}^{2}(1-\tilde{\beta})^{2}\right] .
\end{aligned}
$$

Now we consider the asymptotics of the topological part in the VEV of the energy-momentum tensor near the string and at large distances. The topological part is finite on the string:

$\left.\left\langle T_{\mu}^{\mu}\right\rangle_{c}\right|_{r=0}=\left[1+2 s_{0}\left(q, \alpha_{0}\right)\right]\left\langle T_{\mu}^{\mu}\right\rangle_{c}^{(0)}$,

where $\left\langle T_{\mu}^{\mu}\right\rangle_{c}^{(0)}$ is given by expressions (4.7) and (4.14) for massive and massless fields, respectively. In the absence of magnetic flux along the string axis, $\alpha_{0}=0$, and in accordance with (2.47) the topological part vanishes on the string. The part $\left\langle T_{\mu}^{\mu}\right\rangle_{s}$ diverges on the string and, hence, it dominates in the total VEV for points near the string, $r \ll L$. In the opposite limit, $r \gg L$, and for a massless field, in (4.11) the argument of the function $C(\tilde{\beta}, x)$ is large, $x \gg 1$. In this case we use the asymptotic formula $C(\tilde{\beta}, x) \approx \pi^{2} \sigma e^{-2 \pi \sigma x} /\left(2 x^{2}\right)$, with $\sigma=\min (\tilde{\beta}, 1-\tilde{\beta})$ and $0<\tilde{\beta}<1$. For $\tilde{\beta}=0$ one has $C(0, x) \approx \pi /\left(4 x^{3}\right), x \gg 1$. For $q>2$ and $0<\tilde{\beta}<1$, the dominant contribution to the second term in the right-hand side of (4.11) comes from the $k=1$ term and we have

$\left\langle T_{\mu}^{\mu}\right\rangle \approx\left\langle T_{\mu}^{\mu}\right\rangle_{c}^{(0)}-\frac{\delta_{\mu} \sigma \cos (\pi / q) \cos \left(2 \pi \alpha_{0}\right)}{L^{2} r^{2} \sin ^{2}(\pi / q)} e^{-4 \pi \sigma r \sin (\pi / q) / L}$.

For $q \leqslant 2$, in (4.11) the sum over $k$ is absent and the contribution of the integral term is suppressed by the factor $e^{-4 \pi \sigma r / L}$. Hence, in the case $q \leqslant 2$ the suppression of the effects induced by the string at large distances is stronger. For $\tilde{\beta}=0$ we have the asymptotic formula

$\left\langle T_{\mu}^{\mu}\right\rangle \approx\left\langle T_{\mu}^{\mu}\right\rangle_{c}^{(0)}+\frac{\delta_{\mu} s_{3}\left(q, \alpha_{0}\right)}{4 \pi L r^{3}}$,

with the function $s_{n}\left(q, \alpha_{0}\right)$ defined in (2.46).

In Fig. 5 we have presented the quantity $L^{4}\left\langle T_{0}^{0}\right\rangle$ for a massless fermionic field as a function of the parameters $\alpha_{0}$ and $\tilde{\beta}$ for the fixed values $q=2.5$ and $r / L=0.25$. The dependence of the same function on the distance from the string is displayed in Fig. 6 for $\alpha_{0}=\tilde{\beta}=0.5$. The numbers near the curves correspond to the value of the parameter $q$.

From the discussion given above it follows that we can decompose the VEV of the energy density as

$\left\langle T_{0}^{0}\right\rangle=\left\langle T_{0}^{0}\right\rangle_{c}^{(0)}+\left\langle T_{0}^{0}\right\rangle_{s}^{\text {ren }}+\left\langle T_{0}^{0}\right\rangle_{c}^{(s)}$,

where the last term is the contribution of the cosmic string to the topological part to the energy density. The distribution of the energy density $\left\langle T_{0}^{0}\right\rangle_{c}^{(0)}$, corresponding to the Minkowski

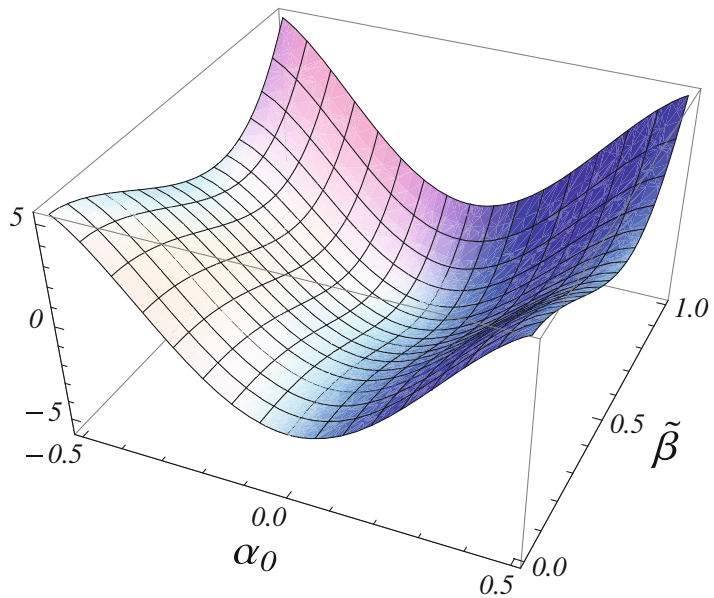

Fig. 5 Energy density, $L^{4}\left\langle T_{0}^{0}\right\rangle$, for a massless fermionic field as a function of $\alpha_{0}$ and $\tilde{\beta}$ for fixed values $q=2.5$ and $r / L=0.25$

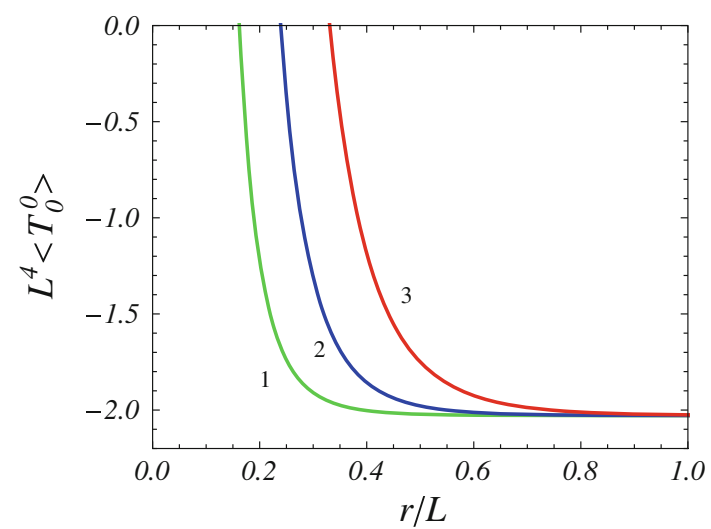

Fig. 6 Energy density, $L^{4}\left\langle T_{0}^{0}\right\rangle$, for a massless fermionic field as a function of the distance from the string for $\alpha_{0}=\tilde{\beta}=0.5$. The numbers near the curves correspond to the value of the parameter $q$

spacetime compactified along the $z$-direction, is uniform. The part $\left\langle T_{0}^{0}\right\rangle_{s}^{\text {ren }}$ behaves near the string as $1 / r^{4}$ and, hence, the corresponding contribution to the vacuum energy is divergent. We can evaluate the total vacuum energy (per unit length of the string) induced by the straight string in the region $r \geqslant r_{0}>0$ :

$E_{s, r \geqslant r_{0}}=\phi_{0} \int_{r_{0}}^{\infty} \mathrm{d} r r\left\langle T_{0}^{0}\right\rangle_{s}^{\text {ren }}$

By using the formula $\int_{a}^{\infty} \mathrm{d} x x f_{v}(x)=f_{v-1}(a)$ and (4.2), one gets

$$
\begin{aligned}
E_{s, r \geqslant r_{0}}= & \frac{m^{2}}{\pi^{2}}\left[\frac{\pi}{q} \sum_{k=1}^{p} \frac{(-1)^{k}}{s_{k}^{2}} \cos (\pi k / q) \cos \left(2 \pi k \alpha_{0}\right) f_{1}\left(2 m r_{0} s_{k}\right)\right. \\
& \left.+\int_{0}^{\infty} \mathrm{d} x \frac{h\left(q, \alpha_{0}, x\right) \sinh x}{\cosh (2 q x)-\cos (q \pi)} \frac{f_{1}\left(2 m r_{0} \cosh x\right)}{\cosh ^{2} x}\right] .
\end{aligned}
$$


For a massless field this expression is written as

$E_{S, r \geqslant r_{0}}=\frac{s_{4}\left(q, \alpha_{0}\right)}{4 \pi^{2} r_{0}^{2}}$.

In the absence of magnetic flux, $\alpha_{0}=0$, this formula is further simplified by using (2.47).

The last term in (4.18) is given by the part in (4.9) with $l \neq 0$. For the corresponding contribution to the total vacuum energy one has

$$
E_{c}^{s}=\int_{0}^{L} \mathrm{~d} z \int_{0}^{\phi_{0}} \mathrm{~d} \phi \int_{0}^{\infty} \mathrm{d} r r\left\langle T_{0}^{0}\right\rangle_{c}^{(s)}=\frac{2 s_{2}\left(q, \alpha_{0}\right)}{\pi q L} F(\tilde{\beta}, m L),
$$

where the function $s_{n}\left(q, \alpha_{0}\right)$ is given by the expression (2.46) and we have defined the function

$F(\tilde{\beta}, x)=x \sum_{l=1}^{\infty} \frac{\cos (2 \pi l \tilde{\beta})}{l} K_{1}(l x)$.

For a massless field the expression for $E_{c}^{s}$ is obtained from (4.22), by taking into account that $F(\tilde{\beta}, 0)=\pi^{2}\left(1 / 6+\tilde{\beta}^{2}-\right.$ $\tilde{\beta})$. In the absence of magnetic flux along the axis of the string one has $\alpha_{0}=0$ and, by using (2.47), one finds

$E_{c}^{s}=\frac{1-q^{2}}{6 \pi q L} F(\tilde{\beta}, m L)$.

In particular, in the absence of magnetic flux enclosed by the compact dimension, $\Phi_{z}=0$, this energy is negative for an untwisted fermionic field $(\beta=0)$ and positive for twisted field $(\beta=1 / 2)$.

\section{Conclusion}

In this paper we have investigated the FC and the VEV of the energy-momentum tensor for a charged massive fermion field in the compactified cosmic string spacetime considering the presence of a constant vector potential. Though the magnetic field strength is zero, the nontrivial spatial topology gives arise to Aharonov-Bohm-like effects on the VEVs. We have assumed that the field operator obeys a quasiperiodicity condition along the $z$-axis with the period $L$, exhibited in (2.6). The phase under this condition and the component of the gauge field along the string axis are related through a gauge transformation and the VEVs depend on the combination (2.13). By applying the Abel-Plana summation formula to the series over the quantum number corresponding to the compact dimension, we have explicitly extracted from the VEVs the contributions corresponding to the geometry of a straight cosmic string. The compactification does not change the local geometry, so the renormalization of the VEVs is reduced to the one for the straight cosmic string spacetime part. Outside the string core the background geometry is flat and for the renormalization we need just to subtract the parts corresponding to the topologically trivial Minkowski spacetime in the absence of magnetic fluxes. The application of the formula (2.28) allowed us to explicitly separate these parts. In this way, we have provided closed analytic expressions for both renormalized straight cosmic string and topological parts as functions of the planar angle deficit and two kinds of magnetic fluxes: flux running along the string axis and flux enclosed by the compact dimension. In particular, both FC and the vacuum energy-momentum tensor are even periodic functions of these fluxes with period equal to the flux quantum.

The renormalized FC in the geometry of a straight cosmic string is given by the expression (2.32). With respect to the dependence on the magnetic flux running along the string axis, the FC can be either positive or negative for a massive field and it vanishes for a massless field. In particular, in the absence of magnetic flux the $\mathrm{FC}$ is positive. The straight cosmic string part in the FC diverges on the string as $1 / r^{2}$. At distances from the string larger than the Compton wavelength of the fermionic particle, it decays as $e^{-2 m r}$ for $1 \leqslant q \leqslant 2$ and as $e^{-2 m r \sin (\pi / q)}$ for $q>2$. The part in the FC induced by the compactification of the cosmic string axis is given by (2.41) (an alternative expression is provided by (2.40)). The $k=0$ term in this expression, see (2.44), corresponds to the FC in Minkowski spacetime with spatial topology $R^{2} \times S^{1}$, denoted as $\langle\bar{\psi} \psi\rangle_{c}^{(0)}$. The topological part is finite on the string and vanishes in the absence of magnetic flux along the string axis. For points near the string, the dominant contribution to the total FC comes from the straight cosmic string part, $\langle\bar{\psi} \psi\rangle_{s}^{\text {ren }}$. On the other hand, at large distances the effects induced by the string decay exponentially and the FC tends to the limiting value which coincides with $\langle\bar{\psi} \psi\rangle_{c}^{(0)}$. For small values of the length of the compact dimension, the asymptotic behavior of the topological part in the FC crucially depends on the values of the parameter $\tilde{\beta}$ describing the magnetic flux enclosed by the compact dimension, i.e. whether it is zero or not. For $0<\tilde{\beta}<1$, the effects of the cosmic string in the topological part of the $\mathrm{FC}$ are suppressed by the factor $\exp [-4 \pi \tilde{\beta} r \sin (\pi / q) / L]$ for $q>2$ and by the factor $\exp (-4 \pi \tilde{\beta} r / L)$ for $1 \leqslant q \leqslant 2$. In the case $\tilde{\beta}=0$ and for small values of $L$, the asymptotic expression for the FC is given by $(2.50)$, where $\langle\bar{\psi} \psi\rangle_{s}^{(D=2)}$ is the FC in a $(2+1)$ dimensional conical spacetime. The numerical calculations have shown that, at a given point, both the sign and the absolute value of the $\mathrm{FC}$ can be effectively tuned by changing the magnetic fluxes.

Another important characteristic of the fermionic vacuum is the VEV of the energy-momentum tensor. The main steps 
of the evaluation for the separate components of this tensor are presented in Sect. 3. Combined expressions for both straight cosmic string and topological parts and also their properties are presented in Sect. 4; in this way we have explicitly shown that these parts obey, separately, the trace relation (4.1) and covariant conservation equation. In particular, the vacuum energy-momentum tensor is traceless for a massless fermionic field. The renormalized VEV of the energymomentum tensor in the geometry of a straight cosmic string is given in a general form by (4.2). This expression includes various special cases previously described in the literature. The radial and axial stresses are equal to the energy density. For the axial stress this result could be directly obtained from the boost invariance along the string axis. For a massless field the general formula is simplified to (4.4). In the absence of magnetic flux we recover the well-known result derived in [30-32]. In the general case of a massive field, near the string the straight cosmic string part behaves as $1 / r^{4}$, and it dominates in the total VEV of the energy-momentum tensor. At large distances from the string, $m r \gg 1$, the leading terms in the corresponding asymptotic expansions are given by (4.6). Note that in this limit $\left|\left\langle T_{0}^{0}\right\rangle_{s}^{\text {ren }}\right| \ll\left|\left\langle T_{\phi}^{\phi}\right\rangle_{s}^{\text {ren }}\right|$. As illustrated in Fig. 4, with a dependence on the magnetic flux, the vacuum energy density in the geometry of a straight cosmic string can be either positive or negative. In particular, the energy density is negative in the absence of magnetic flux.

In the compactified cosmic string spacetime, the total VEV of the energy-momentum tensor is given by (4.9). The term $l=0$ in this expression corresponds to the VEV for the geometry of a straight cosmic string and the remaining part is induced by the compactification of the string axis. The term $\left\langle T_{\mu}^{\mu}\right\rangle_{c}^{(0)}$, defined in (4.7), represents the VEV in Minkowski spacetime with spatial topology $R^{2} \times S^{1}$ in the absence of the cosmic string and flux along the $z$-axis. Alternative expressions for the topological parts in the VEVs are given by (3.12), (3.41), and (3.52). As in the case of the straight cosmic string geometry, the radial stress is equal to the energy density. However, the compactification breaks the boost invariance along the string axis and the axial stress differs from the energy density. Simpler expressions are obtained for two special cases. For integer values of the parameter $q$ and for $\alpha_{0}$ given by (2.30), the integral term vanishes, and we get the expression (4.10). For a massless field the VEV of the energy-momentum tensor is reduced to (4.11). In this special case, the axial stress is equal to the energy density. The topological part in the VEV of the energy-momentum tensor is finite on the string with the limiting value given by (4.15). In the absence of magnetic flux along the string axis the topological part vanishes on the string. At large distances from the string and for a massless field, the part in the VEV of the energy-momentum tensor induced by the string is suppressed by the factor $e^{-4 \pi \sigma r \sin (\pi / q) / L}$ (see (4.16)) for $q>2$ and by $e^{-4 \pi \sigma r / L}$ for $q \leqslant 2$ with $0<\tilde{\beta}<1$ and $\sigma=$ $\min (\tilde{\beta}, 1-\tilde{\beta})$. For $\tilde{\beta}=0$ we have a power-law decay given by (4.17).

In addition to the local characteristics of the vacuum state, we have also evaluated the part in the topological Casimir energy induced by the string. The latter is given by the simple formula (4.22). In the absence of magnetic flux along the axis of the string this formula is further simplified to (4.24). If, in addition, there is no magnetic flux enclosed by the compact dimension, the corresponding energy is negative/positive for untwisted/twisted fermionic fields. The sign of the vacuum energy can be controlled by tuning the values of the magnetic fluxes.

Acknowledgments ERBM thanks Conselho Nacional de Desenvolvimento Científico e Tecnol ógico $(\mathrm{CNPq})$ for partial financial support. AAS was supported by State Committee Science MES RA, within the frame of the research project No. SCS 13-1C040.

Open Access This article is distributed under the terms of the Creative Commons Attribution License which permits any use, distribution, and reproduction in any medium, provided the original author(s) and the source are credited.

Article funded by SCOAP ${ }^{3}$ and licensed under CC BY 4.0

\section{References}

1. E. Elizalde, S.D. Odintsov, A. Romeo, A.A. Bytsenko, S. Zerbini, Zeta Regularization Techniques with Applications (World Scientific, Singapore, 1994)

2. V.M. Mostepanenko, N.N. Trunov, The Casimir Effect and Its Applications (Clarendon, Oxford, 1997)

3. K.A. Milton, The Casimir Effect: Physical Manifestation of ZeroPoint Energy (World Scientific, Singapore, 2002)

4. M. Bordag, G.L. Klimchitskaya, U. Mohideen, V.M. Mostepanenko, Advances in the Casimir Effect (Oxford University Press, Oxford, 2009)

5. M. Bordag, G.L. Klimchitskaya, U. Mohideen, V.M. Mostepanenko, in Lecture Notes in Physics: Casimir Physics, vol. 834, ed. by D. Dalvit, P. Milonni, D. Roberts, F. da Rosa (Springer, Berlin, 2011)

6. E. Elizalde, Phys. Lett. B 516, 143 (2001)

7. C.L. Gardner, Phys. Lett. B 524, 21 (2002)

8. K.A. Milton, Grav. Cosmol. 9, 66 (2003)

9. A.A. Saharian, Phys. Rev. D 70, 064026 (2004)

10. E. Elizalde, J. Phys. A 39, 6299 (2006)

11. A.A. Saharian, Phys. Rev. D 74, 124009 (2006)

12. B. Green, J. Levin, J. High Energy Phys. 11, 096 (2007)

13. P. Burikham, A. Chatrabhuti, P. Patcharamaneepakorn, K. Pimsamarn, J. High Energy Phys. 07, 013 (2008)

14. A.R. Zhitnitsky, Phys. Rev. D 86, 045026 (2012)

15. S. Bellucci, A.A. Saharian, Phys. Rev. D 79, 085019 (2009)

16. E. Elizalde, S.D. Odintsov, A.A. Saharian, Phys. Rev. D 83, 105023 (2011)

17. E.R. Bezerra de Mello, A.A. Saharian, Eur. Phys. J. C. 73, 2532 (2013)

18. E.R. Bezerra de Mello, A.A. Saharian, Class. Quantum Grav. 29, 035006 (2012)

19. T.W.B. Kibble, Phys. Rep. 67, 183 (1980)

20. A. Vilenkin, E.P.S. Shellard, Cosmic Strings and Other Topological Defects (Cambridge University Press, Cambridge, 1994) 
21. V. Berezinski, B. Hnatyk, A. Vilenkin, Phys. Rev. D 64, 043004 (2001)

22. T. Damour, A. Vilenkin, Phys. Rev. Lett. 85, 3761 (2000)

23. P. Bhattacharjee, G. Sig1, Phys. Rep. 327, 109 (2000)

24. S. Sarangi, S.-H. Henry Tye, Phys. Lett. B 536, 185 (2002)

25. E.J. Copeland, R.C. Myers, J. Polchinski, J. High Energy Phys. 06, 013 (2004)

26. G. Dvali, A. Vilenkin, J. Cosmol. Astropart. Phys. 03, 010 (2004)

27. T.W.B. Kibble, arXiv:astro-ph/0410073

28. T.M. Helliwell, D.A. Konkowski, Phys. Rev. D 34, 1918 (1986)

29. B. Linet, Phys. Rev. D 35, 536 (1987)

30. V.P. Frolov, E.M. Serebriany, Phys. Rev. D 35, 3779 (1987)

31. J.S. Dowker, Phys. Rev. D 36, 3095 (1987)

32. J.S. Dowker, Phys. Rev. D 36, 3742 (1987)

33. P.C.W. Davies, V. Sahni, Class. Quantum Grav. 5, 1 (1988)

34. A.G. Smith, in The Formation and Evolution of Cosmic Strings, ed. by G.W. Gibbons, S.W. Hawking, T. Vachaspati. Proceedings of the Cambridge Workshop, Cambridge, England, 1989 (Cambridge University Press, Cambridge, England, 1990)

35. B. Allen, A.C. Ottewill, Phys. Rev. D 42, 2669 (1990)

36. B. Allen, J.G. Mc Laughlin, A.C. Ottewill, Phys. Rev. D 45, 4486 (1992)

37. B. Allen, B.S. Kay, A.C. Ottewill, Phys. Rev. D 53, 6829 (1996)

38. T. Souradeep, V. Sahni, Phys. Rev. D 46, 1616 (1992)

39. K. Shiraishi, S. Hirenzaki, Class. Quantum Grav. 9, 2277 (1992)

40. V.B. Bezerra, E.R. Bezerra de Mello, Class. Quantum Grav. 11, 457 (1994)

41. E.R. Bezerra de Mello, Class. Quantum Grav. 11, 1415 (1994)

42. G. Cognola, K. Kirsten, L. Vanzo, Phys. Rev. D 49, 1029 (1994)

43. E.S. Moreira, J. Nucl. Phys. B 451, 365 (1995)

44. D. Iellici, Class. Quantum Grav. 14, 3287 (1997)

45. N.R. Khusnutdinov, M. Bordag, Phys. Rev. D 59, 064017 (1999)

46. V.B. Bezerra, N.R. Khusnutdinov, Class. Quantum Grav. 23, 3449 (2006)

47. V.M. Bardeghyan, A.A. Saharian, J. Contemp. Phys. (Arm. Acad. Sci.) 45, 1 (2010)

48. A.A. Saharian, A.S. Kotanjyan, Eur. Phys. J. C 71, 1765 (2011)

49. E.R. Bezerra de Mello, V.B. Bezerra, H.F. Mota, A.A. Saharian, Phys. Rev. D 86, 065023 (2012)

50. M.E.X. Guimarães, B. Linet, Commun. Math. Phys. 165, 297 (1994)

51. J. Spinelly, E.R. Bezerra de Mello, Class. Quantum Grav. 20, 874 (2003)
52. J. Spinelly, E.R. Bezerra de Mello, Int. J. Mod. Phys. A 17, 4375 (2002)

53. J. Spinelly, E.R. Bezerra de Mello, Int. J. Mod. Phys. D 13, 607 (2004)

54. J. Spinelly, E.R. Bezerra de Mello, Nucl Phys. B (Proc. Suppl.) 127, 77 (2004)

55. J. Spinelly, E.R. Bezerra de Mello, JHEP 09, 005 (2008)

56. L. Sriramkumar, Class. Quantum Grav. 18, 1015 (2001)

57. YuA Sitenko, N.D. Vlasii, Class. Quantum Grav. 26, 195009 (2009)

58. E.R. Bezerra de Mello, Class. Quantum Grav. 27, 095017 (2010)

59. E.R. Bezerra de Mello, V.B. Bezerra, A.A. Saharian, V.M. Bardeghyan, Phys. Rev. D 82, 085033 (2010)

60. I. Brevik, T. Toverud, Class. Quantum Grav. 12, 1229 (1995)

61. E.R. Bezerra de Mello, V.B. Bezerra, A.A. Saharian, A.S. Tarloyan, Phys. Rev. D 74, 025017 (2006)

62. E.R. Bezerra de Mello, V.B. Bezerra, A.A. Saharian, Phys. Lett. B 645, 245 (2007)

63. E.R. Bezerra de Mello, V.B. Bezerra, A.A. Saharian, A.S. Tarloyan, Phys. Rev. D 78, 105007 (2008)

64. G. Fucci, K. Kirsten, JHEP 1103, 016 (2011)

65. E.R. Bezerra de Mello, A.A. Saharian, Class. Quantum Grav. 28, 145008 (2011)

66. G. Fucci, K. Kirsten, J. Phys. A 44, 295403 (2011)

67. S. Bellucci, E.R. Bezerra de Mello, A.A. Saharian, Phys. Rev. D 83, 085017 (2011)

68. V.V. Nesterenko, I.G. Pirozhenko, Class. Quantum Grav. 28, 175020 (2011)

69. E.R. Bezerra de Mello, F. Moraes, A.A. Saharian, Phys. Rev. D 85, $045016(2012)$

70. E.R. Bezerra de Mello, A.A. Saharian, AKh Grigoryan, J. Phys. A Math. Theor. 45, 374011 (2012)

71. E.R. Bezerra de Mell, A.A. Saharian, S.V. Abajyan, Class. Quantum Grav. 30, 015002 (2013)

72. S. Bellucci, A.A. Saharian, V.M. Bardeghyan, Phys. Rev. D 82, $065011(2010)$

73. A.A. Saharian, The Generalized Abel-Plana Formula with Applications to Bessel Functions and Casimir Effect (Yerevan State University Publishing House, Yerevan, 2008). Report No. ICTP/2007/082; arXiv:0708.1187

74. I.S. Gradshteyn, I.M. Ryzhik, Table of Integrals, Series and Products (Academic Press, New York, 1980)

75. A.P. Prudnikov, Yu. A. Brychkov, O.I. Marichev, Integrals and Series, vol. 1 (Gordon and Breach, New York, 1986) 